\title{
The Spectral Class of the Quantum-Mechanical Harmonic Oscillator
}

\author{
H. P. McKean and E. Trubowitz*
}

Courant Institute of Mathematical Sciences, New York University, New York, NY 10012, USA

\begin{abstract}
The purpose of this paper is to study the so-called spectral class $\mathbf{Q}$ of anharmonic oscillators $Q=-D^{2}+q$ having the same spectrum $\lambda_{n}=2 n$ $(n \geqq 0)$ as the harmonic oscillator $Q^{0}=-D^{2}+x^{2}-1$. The norming constants $t_{n}=\lim _{x \uparrow \infty} \ell \mathrm{g}\left[(-1)^{n} e_{n}(x) / e_{n}(-x)\right]$ of the eigenfunctions of $Q$ form a complete set of coordinates in $\mathbf{Q}$ in terms of which the potential may be expressed as $q=x^{2}-1-2 D^{2} \ell \mathrm{g} \theta$ with

$$
\theta=\operatorname{det}\left[\delta_{i j}+\left(e^{t_{\imath}}-1\right) \int_{x}^{\infty} e_{i}^{0} e_{j}^{0}: 0 \leqq i, j,<\infty\right],
$$

$e_{n}^{0}$ being the $n^{\text {th }}$ eigenfunction $Q^{0}$. The spectrum and norming constants are canonically conjugate relative to the bracket $[F, G]=\int \nabla F D \nabla G d x$, to wit: $\left[\lambda_{i}, \lambda_{j}\right]=0,\left[t_{i}, 2 \lambda_{j}\right]=1$ or 0 according to whether $i=j$ or not, and $\left[t_{i}, t_{j}\right]=0$. This prompts an investigation of the symplectic geometry of $\mathbf{Q}$. The function $\theta$ is related to the theta function of a singular algebraic curve. Numerical results are also presented.
\end{abstract}

\section{Introduction}

The spectrum of the quantum-mechanical harmonic oscillator ${ }^{1} Q^{0}=-D^{2}+$ $x^{2}-1$ is $0,2,4,6$, etc. The corresponding unit eigenfunctions are the Hermite functions:

$$
e_{n}^{0}(x)=\left(\sqrt{\pi} 2^{n} n !\right)^{-1 / 2} e^{x^{2} / 2} D^{n} e^{-x^{2}} \quad(n \geqq 0) .
$$

Let $\Delta q$ belong to the class $\mathbf{S}(\mathbf{R})$ of real infinitely differentiable functions vanishing rapidly at $\pm \infty^{2}$. The anharmonic oscillator $Q=-D^{2}+q$ with potential $q=x^{2}-1+\Delta q$ has a discrete spectrum of simple eigenvalues $\lambda_{n}=\lambda_{n}[q]$, increasing to $+\infty$ with $n$, and corresponding unit eigenfunctions $e_{n}(n \geqq 0)$ of class $\mathbf{S}$. The

\footnotetext{
* Sloan Foundation Fellow.

$1 D$ signifies differentiation with regard to $x$.

$2 x^{i} D^{j} \Delta q=o(1)$ for $x \rightarrow \pm \infty$ and every $i, j \geqq 0$.
} 
purpose of this paper is to study the spectral class $\mathbf{Q}=\mathbf{Q}\left[x^{2}-1\right]$ of such oscillators having the same spectrum $\lambda_{n}=\lambda_{n}^{0}=2 n$ as $Q^{0}$, i.e., the aim is to explain to what extent the quantum-mechanical oscillator is specified by its spectrum. The principal results and their geometrical motivation will now be described.

\section{Isospectral Flows}

The flow of translation $\partial q / \partial t=\partial q / \partial x$ leads immediately out of the class $x^{2}-1+\mathbf{S}$; more drastically, the KDV flow $\partial q / \partial t=3 q \partial q / \partial x-(1 / 2) \partial^{3} q / \partial x^{3}$ does not even exist for $q \sim x^{2}$ as the individual terms cannot balance unless $q$ is sublinear. Fortunately, a wide class of isospectral flows suggests itself by elementary geometrical reasoning: $\mathbf{Q}$ is defined by the relations $\lambda_{n}=2 n(n \geqq 0)$, so the normal space to $\mathbf{Q}$ at $q$ is, or ought to be, the span of the gradients ${ }^{3} \nabla \lambda_{n}=e_{n}^{2}(n \geqq 0)$. Now $D e_{i}^{2}$ is perpendicular to $e_{j}^{2}$ for every $i$ and $j$, as is plain for $i=j$, while for $i \neq j$, it follows from $^{4}$

$$
D\left[e_{i}, e_{j}\right]=e_{i} e_{j}^{\prime \prime}-e_{i}^{\prime \prime} e_{j}=\left(\lambda_{i}-\lambda_{j}\right) e_{i} e_{j}
$$

and

$$
\begin{aligned}
\int e_{i}^{2} D e_{j}^{2} & =-\int e_{j}^{2} D e_{i}^{2}=\frac{1}{2} \int\left[e_{i}^{2} D e_{j}^{2}-e_{j}^{2} D e_{i}^{2}\right] \\
& =\int e_{i} e_{j}\left[e_{i}, e_{j}\right]=\left.\left(\lambda_{i}-\lambda_{j}\right)^{-1} \frac{1}{2}\left[e_{i}, e_{j}\right]^{2}\right|_{-\infty} ^{\infty}=0 .
\end{aligned}
$$

This suggests that the tangent space to $\mathbf{Q}$ at $q$ ought to be the span of $D e_{n}^{2}(n \geqq 0)$ and that ${ }^{5} \partial q / \partial t=2 D e_{n}^{2}=\mathbf{X}_{n} q$ ought to be an isospectral flow. The perpendicularity of $e_{i}^{2}$ and $D e_{j}^{2}$ can be phrased more elegantly in terms of the Poisson bracket, ${ }^{6}$ $[F, G]=\int \nabla F D \nabla G d x$, as $\left[\lambda_{i}, \lambda_{j}\right]=0$. This states that the eigenvalues are involutive and suggests that the flows $\partial q / \partial t=\mathbf{X} q$ ought to commute. It is a source of satisfaction that $e_{i}^{2}$ and $D e_{j}^{2}$ span $\mathbf{L}^{2}(\mathbf{R})$ so that no direction at $q$ is left unclassified; also, the gradients $\nabla \lambda_{n}=e_{n}^{2}$ of the relations $\lambda_{n}=2 n$ are highly independent in the sense that no direction in the span of some subclass lies in the span of the complementary class [see Sect. 4]. This indicates that $\mathbf{Q}$ is a smooth submanifold of the ambient space $x^{2}-1+\mathbf{S}$, though the point is not pursued below.

\section{The Exponential Map}

It is a pleasant fact that the flows $\partial q / \partial t=2 D e_{n}^{2}$ may be integrated in a simple and explicit manner, obviating any discussion of existence and the like. Let $\mathbf{X}_{n}$ be the vector field $q \rightarrow 2 D e_{n}^{2}$ and fix $q^{0}$ in $\mathbf{Q}\left[x^{2}-1\right]$, not necessarily at $q^{0}(x)=x^{2}-1$. Then the flow $\partial q / \partial t=\mathbf{X}_{n} q$ originating at $q=q^{0}$ is expressed by

$$
q=q^{0}-2 D^{2} \ell \mathrm{g} \theta
$$

$3 \nabla$ is the gradient in function space. The evaluation is elementary from the simplicity of the eigenvalue, the variational equation $Q e_{n}^{\cdot}+\dot{q} e_{n}=\lambda_{n} e_{n}+\lambda_{n} e_{n}^{\cdot}$, and the fact that $\int e_{n} e_{n}^{\cdot}=0$ in view of $\int e_{n}^{2}=1$.

$4\left[e_{i}, e_{j}\right] \equiv e_{i} e_{j}^{\prime}-e_{i}^{\prime} e_{j}$.

5 The factor 2 is introduced with a view to the simplicity of subsequent formulas.

$6[F, G]$ is skew-symmetric and satisfies Jacobı's identity; see, for example, McKean-Moerbeke $[1975]$. 
with

$$
\theta=1+\left(e^{t}-1\right) \int_{x}^{\infty}\left(e_{n}^{0}\right)^{2}
$$

$e_{n}^{0}$ being the initial eigenfunction $\left[Q^{0} e_{n}^{0}=\lambda_{n} e_{n}^{0}\right]$. More generally, let the numbers $t_{n}(n \geqq 0)$ vanish rapidly as $n \uparrow \infty$ and let $\mathbf{X}=\Sigma t_{n} \mathbf{X}_{n}$. Then $e^{\mathbf{X}} q^{0}$ belongs to $\mathbf{Q}$ and may be expressed by the same formula with ${ }^{7}$

$$
\theta=\operatorname{det}\left[\delta_{i j}+\left(e^{t_{i}}-1\right) \int_{x}^{\infty} e_{i}^{0} e_{j}^{0}: 0 \leqq i, j<\infty\right] .
$$

The numbers $t_{n}$ have the interpretation of norming constants: $c_{n}^{-}=e_{n} / e_{n}^{0}(-\infty)$ and $c_{n}^{+}=e_{n} / e_{n}^{0}(+\infty)$ exist and satisfy the connection rule $c_{n}^{-} c_{n}^{+}=1$, and $t_{n}=$ $\ell \mathrm{g} c_{n}^{+} / c_{n}^{-}$; in particular, if $q^{0}(x)=x^{2}-1$, as is mostly the preferred choice, then the map $t \rightarrow-t$ corresponds to the reflection of potentials $q(x) \rightarrow q(-x)$. The geometrical content of all this is that the formula $e^{\mathbf{x}} q^{0}=q^{0}-2 D^{2} \ell \mathrm{g} \theta$ establishes an exponential map from the tangent space of $\mathbf{Q}$ at $q^{0}$ into $\mathbf{Q}$ : it is $1: 1$ in view of the meaning of the parameters $t_{n}$, and in fact it is onto so that $t$ is a global coordinate on $\mathbf{Q}$, relative, e.g., to the origin $q^{0}(x)=x^{2}-1$; in particular, $x^{2}-1$ is the only even potential in its class. The first fundamental form of $\mathbf{Q}$ at $q$ is $g_{i j}=4 \int D e_{i}^{2} D e_{j}^{2}$, i.e., $\|d q\|_{2}^{2}=\sum g_{i j} d t_{i} d t_{j}$. This is a complicated animal even at the origin $q^{0}=x^{2}-1$, and no attempt is made to deal with it beyond noting the formula

$$
\sum g_{i j}^{0} a^{i} b^{j}=4(2 \pi)^{-1 / 2}(1-a)^{1 / 2}(1-b)^{1 / 2}(1-a b)^{-1 / 2}(0 \leqq a, b<1) .
$$

\section{Symplectic Geometry}

The quantities $\lambda_{j}(j \geqq 0)$ are involutive relative to the bracket $[F, G]$, as noted before. It turns out that so are quantities $t_{i}=\lim _{x \uparrow \infty} \ell \mathrm{g}\left[(-1)^{i} e_{i}(x) / e_{i}(-x)\right]$, and the fact that the flow $\partial q / \partial t=\mathbf{X}_{j} q=\left[q, 2 \lambda_{j}\right]$ advances $t_{i}$ at speed 1 if $i=j$, and not at all if $i \neq j$, is to say that $t_{i}(i \geqq 0)$ and $2 \lambda_{j}(j \geqq 0)$ are canonically conjugate relative to the bracket:

$$
\left[t_{i}, 2 \lambda_{j}\right]=1(i=j)=0(i \neq j) .
$$

A more global viewpoint is now adopted. The involutive quantities $t_{n}$ have an existence outside $\mathbf{Q}\left[x^{2}-1\right]$ and so produce commuting vector fields $\mathbf{Y}_{n}: q \rightarrow D \nabla t_{n}$ transversal to the isospectral fields envisaged before, leading off $\mathbf{Q}\left[x^{2}-1\right]$ into the ambient space; they fix the $t_{i}$ 's and move the $\lambda_{j}$ 's. It turns out that $x^{2}-1+\mathbf{S}$ is too small an ambient space for the individual flows, but if $\mathbf{S}$ is appropriately enlarged things appear to proceed nicely. The ambient space is now cut up by two transverse foliations: one foliation has leaves $\mathbf{Q}$ defined by fixing $\lambda_{n}(n \geqq 0)$ not at the eigenvalues $\lambda_{n}\left[x^{2}-1\right]=2 n$, but at some other values with realistic comportment as $n \uparrow \infty$. The typical leaf $\mathbf{P}$ of the second foliation is obtained by fixing the numbers $t_{n}(n \geqq 0)$ in a similar fashion. Two leaves $\mathbf{Q}$ and $\mathbf{P}$ meet in a single point,

7 The formula is of Gelfand-Levitan-type; see, especially, Kay-Moses [1956] and Tanaka [1972/73]. 
and the meeting is transversal as expressed by the fact that the corresponding normal spaces, spanned by $\nabla \lambda_{i}$ and $\nabla t_{j}$, meet only in the null function and fill up $\mathbf{L}^{2}$. The isospectral flows $\partial q / \partial t=\mathbf{X}_{n} q$ preserve the leaf $\mathbf{Q}$ and are integrated by the previous rule: $\Delta q=-2 D^{2} \ell \mathrm{g}\left[1+\left(e^{t}-1\right) \int_{x}^{\infty}\left(e_{n}^{0}\right)^{2}\right]$; likewise, the transversal flows $\partial q / \partial t=\mathbf{Y}_{n} q$ preserve the leaf $\mathbf{P}$ and are integrated by a simple rule: $\Delta q=$ $-2 D^{2} \ell \mathrm{g}\left[e_{n}^{0}, f_{n}^{0}\right], f_{n}^{0}$ being any independent eigenfunction of $Q^{0}$ with updated eigenvalue $\lambda_{n}=\lambda_{n}^{0}+t$. The latter flows are of a different kind from the former in that their parameters $\lambda_{n}(n \geqq 0)$ lie on the $\infty$-dimensional simplex $\lambda_{0}<\lambda_{1}<\lambda_{2}<$ etc. so that they have only a circumscribed existence; in this connection, it is amusing to note that the leaf $\mathbf{P}\left[x^{2}-1\right]$ appears to be precisely the class of even potentials and so is perfectly nice, only it is incomplete as regards these flows. This whole global picture is partly conjectural. The technical effort required to confirm it seems disproportionate to the result, so only the leaf $\mathbf{Q}\left[x^{2}-1\right]$ is treated below, though the proofs have a wider applicability [see Sect. 4-5]. To be candid, it is not even plain what the ambient space should be: for example, there exist potentials outside the present class $\mathbf{Q}\left[x^{2}-1\right]$ with spectrum $\lambda_{n}=2 n$ but exhibiting a charge: $\Delta q(\infty)-\Delta q(-\infty)=4$. It is conjectured that this charge is always integral in any enlargement of $\mathbf{Q}\left[x^{2}-1\right]$ and labels its connected pieces.

\section{Theta Functions}

The letter $\theta$ is used to point up the similarity between this determinant and the Riemann-theta function as it appears in the inverse theory of Hill's equation; see, for example, McKean-Trubowitz [1976]. A singular 2-sheeted curve of infinite genus, with singular points $\lambda_{n}=2 n(n \geqq 0)$ or whatever, lies in the background. The associated Jacobi variety splits up into an uncountable number of components indexed by the real number $x$, provided with the family of singular theta sums:

$$
\theta=\theta_{x}\left(t_{0}, t_{1}, t_{2}, \ldots\right)=\operatorname{det}\left[\delta_{i j}+\left(e^{t_{2}}-1\right) \int_{x}^{\infty} e_{i}^{0} e_{j}^{0}: 0 \leqq i, j<\infty\right] .
$$

The numbers $t_{n}(n \geqq 0)$ may even be expressed as sums, over the points of a certain divisor on the curve, of integrals of differentials of the first kind [see Sect. 6].

\section{Numerical Results}

The appendix contains pictures of several potentials from $\mathbf{Q}\left[x^{2}-1\right]$ displaying the effect of the isospectral flows. They were made by O. McBryan by numerical evaluation of $\theta$.

\section{Finite Interval}

The whole situation is similar but technically simpler for operators $Q$ acting, for example, on functions of $-1 \leqq x \leqq 1$ vanishing at $x= \pm 1$. This will be dealt with in detail in a forthcoming publication of E. Trubowitz. 


\section{The Exponential Map}

Let $\mathbf{Q}=\mathbf{Q}\left[x^{2}-1\right]$ be the spectral class of the quantum-mechanical oscillator $Q^{0}=-D^{2}+x^{2}-1$ obtained by fixing the eigenvalues $\lambda_{n}=\lambda_{n}^{0}=2 n(n \geqq 0)$ in the space $x^{2}-1+\mathbf{S}$. It is proposed to make an exponential map of the tangent vector ${ }^{8} \mathbf{X}=\Sigma t_{i} \mathbf{X}_{i}$ into $\mathbf{Q}$ via the rule $e^{X} q^{0}=q^{0}-2 D^{2} \ell \mathrm{g} \theta=q$ with

$$
\theta=\theta_{x}\left(t_{0}, t_{1}, t_{2}, \ldots\right)=\operatorname{det}\left[\delta_{i j}+\left(e^{t_{i}}-1\right) \int_{x}^{\infty} e_{i}^{0} e_{j}^{0}: 0 \leqq i, j<\infty\right] .
$$

The initial potential $q^{0}$ is any point of $\mathbf{Q}$; it is specialized to the origin $x^{2}-1$ later. The numbers $t_{j}$ vanish rapidly as $j \uparrow \infty$. They have the meaning of norming constants:

$$
e_{j} / e_{j}^{0}( \pm \infty)=\exp \left( \pm t_{j} / 2\right) \text {. }
$$

This makes plain that the exponential map is $1: 1$; the proof that it is also onto is postponed to Sect. 3 .

Step 1. The discussion begins with a single parameter $t=t_{n}$ so that $\theta=$ $1+\left(e^{t}-1\right) \int_{x}^{\infty}\left(e_{n}^{0}\right)^{2}$. It is to be proved that $q=q^{0}-2 D^{2} \ell \mathrm{g} \theta$ is an integral curve of the vector field $\mathbf{X}_{n}: q \rightarrow 2 D e_{n}^{2}$, i.e., $\partial q / \partial t=\mathbf{X} q$. The question of uniqueness is routine and may be left aside.

Proof. $\theta$ is positive by inspection, so the recipe makes sense; moreover, $\theta=1$ at $\infty$ and $e^{t}$ at $-\infty$, so $\Delta q=-2 D^{2} \ell \mathrm{g} \theta$ is class $\mathbf{S}$. Now the function $\theta^{-1} e^{t / 2} e_{n}^{0}=f$ is an eigenfunction of $Q=-D^{2}+q$ with eigenvalue $2 n$, by direct computation, and as it has precisely $n$ roots in common with $e_{n}^{0}$ and satisfies

$$
\int f^{2}=e^{t} \int\left(e_{n}^{0}\right)^{2}\left[1+\left(e^{t}-1\right) \int_{x}^{\infty}\left(e_{n}^{0}\right)^{2}\right]^{-2} d x=\left.e^{t}\left(e^{t}-1\right)^{-1} \theta^{-1}\right|_{-\infty} ^{\infty}=1,
$$

it can only be the $n^{\text {th }}$ eigenfunction $e_{n}$ of $Q$. Besides,

$$
\begin{aligned}
\partial q / \partial t & =-2 D^{2} \ell \mathrm{g}[\partial \theta / \partial t]=-2 D^{2} \theta^{-1} e^{t} \int_{x}^{\infty}\left(e_{n}^{0}\right)^{2} \\
& =2 D\left[\theta^{-1} e^{t / 2} e_{n}^{0}\right]^{2}=2 D e_{n}^{2}=\mathbf{X}_{n} q,
\end{aligned}
$$

by elementary computation, and since the flow preserves the spectrum of $Q$ in view of $\lambda_{m}=2\left[\lambda_{m}, \lambda_{n}\right]=0$, so $q$ belongs to $\mathbf{Q}$ and may be identified as stated.

Amplification 1. The other eigenfunctions of $Q$ are

$$
e_{m}=e_{m}^{0}-\theta^{-1}\left(e^{t}-1\right) e_{n}^{0} \int_{x}^{\infty} e_{m}^{0} e_{n}^{0} \quad(m \neq n) .
$$

The computation is facilitated by the identity $\int_{x}^{\infty} e_{m}^{0} e_{n}^{0}=\left(\lambda_{n}^{0}-\lambda_{m}^{0}\right)^{-1}\left[e_{m}^{0}, e_{n}^{0}\right]$.

$8 \quad X_{J}: q \rightarrow D e_{j}^{2}(j \geqq 0)$. 
Amplification 2. The preceding formula leads, after some tears, to the identity

$$
\int_{x}^{\infty} e_{i} e_{j}=\int_{x}^{\infty} e_{i}^{0} e_{j}^{0}-\theta^{-1}\left(e^{t}-1\right) \int_{x}^{\infty} e_{i}^{0} e_{n}^{0} \int_{x}^{\infty} e_{j}^{0} e_{n}^{0} \quad(i, j \neq n) .
$$

This will be used presently.

Step 2 is to prove that

$$
\begin{aligned}
& q^{0}-2 D^{2} \ell \operatorname{g} \theta \\
& \theta=\operatorname{det}\left[\delta_{i j}+\left(e^{t_{\imath}}-1\right) \int_{x}^{\infty} e_{i}^{0} e_{j}^{0}: 0 \leqq i, j \leqq n\right]
\end{aligned}
$$

represents the action of $e^{\mathbf{x}}$ upon $q^{0}$ for any tame combination $\mathbf{X}=\sum_{i \leqq n} t_{i} \mathbf{X}_{i}$ of the indi-
vidual fields. Proof. The formula of Step 1 and the commutativity of the individual flows ${ }^{9}$ imply that $q_{n}=e^{\mathbf{x}} q^{0}$ can be expressed inductively as

$$
q_{n-1}-2 D^{2} \lg \theta_{n}
$$

with $q_{-1}=q^{0}$ and $\theta_{n}=1+\left(e^{t_{n}}-1\right) \int^{\infty}\left(e_{n}^{-}\right)^{2}, e_{n}^{-}$being the $n^{\text {th }}$ eigenfunction of the preceding potential $q_{n-1}{ }^{10}$. Thus, $\Delta q^{x}=q_{n}-q_{-1}$ is $(-2) \times$ the second logarithmic derivative of the product $\prod_{i \leqq n} \theta_{i}$, and it is required to identify the latter as the stated determinant. This is done by Gaussian elimination with the help of Amplification 2: elimination of the first row of the determinant leads to the product of $\theta_{0}$ and

$$
\begin{aligned}
& \operatorname{det}\left[\delta_{i j}+\left(e^{t_{\imath}}-1\right)\left[\int_{x}^{\infty} e_{i}^{0} e_{j}^{0}-\theta_{0}^{-1}\left(e^{t_{0}}-1\right) \int_{x}^{\infty} e_{i}^{0} e_{0}^{0} \int_{x}^{\infty} e_{j}^{0} e_{0}^{0}\right]: 1 \leqq i, j \leqq n\right] \\
& =\operatorname{det}\left[\delta_{i j}+\left(e^{t_{l}}-1\right) \int_{x}^{\infty} e_{i}^{+} e_{j}^{+}: 1 \leqq i, j \leqq n\right]
\end{aligned}
$$

in which $e_{n}^{+}(n \geqq 0)$ are the eigenfunctions of $e^{t_{0} \mathbf{x}_{0}} q_{-1}=q_{0}$. The rule $\theta=\prod_{i \leqq n} \theta_{i}$ follows by induction.

Amplification 3. The product rule for $\theta$ leads to a proof of its existence and nonvanishing for general rapidly vanishing parameters:

$$
\theta=\prod_{n \geqq 0}\left[1+\left(e^{t_{n}}-1\right) \int_{x}^{\infty}\left(e_{n}^{-}\right)^{2}\right]
$$

and

$$
\sum\left|e^{t_{n}}-1\right| \int_{x}^{\infty}\left(e_{n}^{-}\right)^{2} \leqq \sum\left|e^{t_{n}}-1\right|<\infty
$$

independently of $x$.

$9\left[\lambda_{l}, \lambda_{j}\right]=0$ expresses this fact.

10 The abuse of notation is only momentary. 
Amplification 4. The evaluation $\Delta q=-2 D^{2} \ell \mathrm{g} \theta$ and the rule $\partial q / \partial t_{j}=X_{j} q=$ $2 D e_{j}^{2}$ lead to the identity $e_{j}^{2}=-D\left[\partial \ell \mathrm{g} \theta / \partial t_{j}\right](j \geqq 0)$.

Amplification 5. The formula for the individual flows of Step 1 lead inductively to the evaluations

$$
c_{k}^{-}=\left(e_{k} / e_{k}^{0}\right)(-\infty)=e^{-t_{k} / 2}, \quad c_{k}^{+}=\left(e_{k} / e_{k}^{0}\right)(+\infty)=e^{t_{k} / 2},
$$

whence the connection rule $c_{k}^{-} c_{k}^{+}=1$ and the interpretation of $t_{k}=\ell \mathrm{g} c_{k}^{+} / c_{k}^{-}$as a norming constant. The fact that the (tame) exponential map is $1: 1$ is now plain.

Step 3 is to carry Step 2 over from tame to general rapidly vanishing $t_{n}(n \geqq 0)$. Routine estimates based upon the expansion ${ }^{11}$

$$
\begin{aligned}
\theta & =1+\sum_{n=1}^{\infty} \operatorname{sp} \wedge^{n}\left[\left(e^{t_{i}}-1\right) \int_{x}^{\infty} e_{i}^{0} e_{j}^{0}\right] \\
& =1+\sum_{i}\left(e^{t_{i}}-1\right) \Delta\left[e_{i}^{0}\right]+\sum_{i<j}\left(e^{t_{i}}-1\right)\left(e^{t_{j}}-1\right) \Delta\left[e_{i}^{0}, e_{j}^{0}\right]+\text { etc. }
\end{aligned}
$$

show that $\Delta q=-2 D^{2} \ell \mathrm{g} \theta$ is of class $\mathbf{S}$. The isospectrality of $q=q^{0}+\Delta q$ and its identification as $e^{\mathbf{x}} q^{0}$ will be plain, the only moot point being the interpretation of the parameters $t_{n}$ as norming constants, as in Amplification 5. But that is clear from the estimate

$$
\sum_{i \neq j}\left[\left(e^{t_{2}}-1\right) \int_{x}^{\infty} e_{i}^{0} e_{j}^{0}\right]^{2} \leqq \sum_{i}\left(e^{t_{i}}-1\right)^{2} \int_{x}^{\infty}\left(e_{i}^{0}\right)^{2}=o(1) \quad(x \uparrow \infty)
$$

and Amplification $4^{12}$ :

$$
\begin{aligned}
e_{n}^{2} & =-D\left[\partial \ell g \theta / \partial t_{n}\right] \\
& =-D \sum_{i=0}^{\infty}\left[\delta_{i n}+\left(e^{t_{n}}-1\right) \int_{x}^{\infty} e_{i}^{0} e_{n}^{0}\right]^{-1} e^{t_{n}} \int_{x}^{\infty} e_{n}^{0} e_{i}^{0} \\
& =e^{t_{n}}\left(e_{n}^{0}\right)^{2} \times[1+o(1)] \quad(x \uparrow \infty) .
\end{aligned}
$$

Amplification 6. Choose $q^{0}(x)=x^{2}-1$ as the origin of $\mathbf{Q}$. Then the alternately even and odd parity of the Hermite functions $e_{n}^{0}$ implies

$$
\exp \left(\sum t_{n}\right) \theta_{x}\left(-t_{0},-t_{1},-t_{2}, \text { etc. }\right)=\theta_{-x}\left(t_{0}, t_{1}, t_{2}, \text { etc. }\right),
$$

i.e., the map $\mathbf{X} \rightarrow-\mathbf{X}$ expressing reflection in the tangent space is mirrored in the reflection $q(x) \rightarrow q(-x)$ of the potential $q=e^{\mathbf{x}} q^{0}$. The present choice of origin is to be understood until further notice.

\section{Surjection}

The fact that the exponential map is onto the whole of $\mathbf{Q}$ is harder to prove. The $n^{\text {th }}$ eigenfunction $e_{n}$ of a fixed potential $q$ of class $\mathbf{Q}$ is proportional to $x^{n} e^{-x^{2} / 2}$

$11 \Delta\left[f_{1}, \ldots, f_{n}\right]=\operatorname{det}\left[\int_{x}^{\infty} f_{i} f_{j}: 1 \leqq i, j \leqq n\right]$.

12 The exponent -1 in the sum signifies the inverse matrix. The fact that $e_{n}(x)=0$ has precisely $n$ roots must also be used. 
at $\pm \infty$, so the constants

$$
c_{n}^{-}=\left(e_{n} / e_{n}^{0}\right)(-\infty), \quad c_{n}^{+}=\left(e_{n} / e_{n}^{0}\right)(+\infty)
$$

exist. It is required to prove the connection rule $c_{n}^{-} c_{n}^{+}=1$ and the rapid vanishing of $t_{n}=\ell \mathbf{g} c_{n}^{+} / c_{n}^{-}$, and to verify $q=e^{\mathbf{X}} q^{0}$ with $\mathbf{X}=\sum t_{n} \mathbf{X}_{n}$.

\section{Connection Rule}

The first 2 steps are preparatory.

Step 1. Introduce the more-or-less standard Hermite-Weber function:

$$
w_{+}^{0}(x, \lambda)=\Gamma(p) \frac{e^{-x^{2} / 2}}{2 \pi \sqrt{-1}} \int e^{2 x y-y^{2}} y^{-p} d y
$$

with $p=1+\lambda / 2$. The integral is performed about the contour of Fig. 1 and the fractional power $y^{-p}$ is the principal branch in the plane cut along $(-\infty, 0]$. The allied function $w_{-}^{0}(x, \lambda)=w_{+}^{0}(-x, \lambda)$ is also introduced. The following information comes from Bateman [1953: 116 et seq.] :

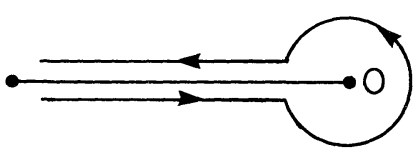

Fig. 1.

a) $Q^{0} w=\lambda w$ for $w=w_{-}^{0}$ or $w_{+}^{0}$.

b) $w_{+}^{0}$ is comparable to $x^{p-1} e^{-x^{2} / 2}$ at $x=\infty$ and to $x^{-p} e^{x^{2} / 2}$ at $x=-\infty$.

c) $w_{+}^{0}$ is an integral function of $\lambda$, of order 1 and maximal type for fixed $x$.

d) $4^{0}(\lambda)=\left[w_{-}^{0}, w_{+}^{0}\right]$ is independent of $x$; it is an integral function of the same class with simple roots at $\lambda=0,2,4,6$ etc. and no others; in particular, for $\lambda=2 n \geqq 0$, both $w_{-}^{0}$ and $w_{+}^{0}$ are proportional to the Hermite function $e_{n}^{0}$.

Step 2. The Hermite-Weber functions of Step 1 may be imitated for general $Q$ with $\Delta q$ vanishing at $\pm \infty$. These new functions are designated $w_{ \pm}(x, \lambda)$ and together with $\Delta(\lambda)=\left[w_{-}, w_{+}\right]$have the same properties a)-d) as their prototypes. The routine discussion is based upon the recipe

$$
w_{+}(x)=w_{+}^{0}(x)^{0}+\int_{x}^{\infty}\left[w_{+}^{0}(x) w_{-}^{0}(y)-w_{-}^{0}(x) w_{+}^{0}(y)\right] w_{+}(y) \Delta q(y) d y
$$

and may be omitted. The comparison $w_{+}=w_{+}^{0}\left[1+0\left(\lambda^{-1 / 2}\right)\right]$, valid for fixed $x$ and $\lambda \downarrow-\infty$, is noted for future use; it may be differentiated by $x$.

Step 3 is the proof of the connection rule: Off spectrum, the Green's function $(Q-\lambda)_{x y}^{-1}$ may be expressed either as $-\Delta^{-1} w_{-}(x) w_{+}(y)(x<y)$ or as the sum 


$$
\begin{aligned}
& \sum\left(\lambda_{n}-x\right)^{-1} e_{n}(x) e_{n}(y), \text { whence }^{13} \\
& {\left[\Delta\left(\lambda_{n}\right)\right]^{-1} w_{-}\left(x, \lambda_{n}\right) w_{+}\left(y, \lambda_{n}\right)=e_{n}(x) e(y) \quad(x<y),}
\end{aligned}
$$

by matching residues at $\lambda=\lambda_{n}$, and $c_{n}^{-} c_{n}^{+}=\Delta^{0} / \Delta^{0}$ at $\lambda=\lambda_{n}$. But $\Delta$ and its prototype $\Delta^{0}$ are integral functions of order 1 vanishing simply at $\lambda_{n}=2 n(n \geqq 0)$, and $\Delta(\lambda) \sim \Delta^{0}(\lambda)$ for $\lambda \downarrow-\infty$ by the comparison of Step 2. $\Delta=\Delta^{0}$ and $c_{n}^{-} c_{n}^{+}=1$ follow: such a function $\Delta$ differs from its Hadamard product only by a factor $e^{a+b \lambda}$ and two such factors coincide if their ratio tends to 1 along a ray.

\section{Uniqueness}

It will be necessary to know that $c_{n}^{+} / c_{n}^{-}=1(n \geqq 0)$ only at the origin of $\mathbf{Q}$, i.e., only if $q(x)=x^{2}-1$. It is a corollary that $x^{2}-1$ is the only even potential of class $\mathbf{Q}$; if $q(x)=q(-x)$, then its eigenfunctions, like those of $q^{0}(x)=x^{2}-1$, are alternately even or odd, so that $c_{n}^{+} / c_{n}^{-}=1$. The proof of uniqueness is modeled upon Levinson [1949] and is the analogue of a theorem of Borg [1945].

Step 1 consists of preliminary estimates. Fix a number $x$ and let $q^{x}(y)$ be $y^{2}-1$ or $q(y)$ according as $y<x$ or $y \geqq x$. The eigenvalues $\lambda_{n}^{x}$ and the eigenfunctions $e_{n}^{x}(y)$ of the corresponding operator $Q^{x}=-d^{2} / d y^{2}+q^{x}$ move with $x$, and it is easy to see that

$$
\frac{\partial \lambda_{n}^{x}}{\partial x}=\left[e_{n}^{x}(x)\right]^{2} \Delta q(x)
$$

Now $^{14}$ for $|y| \leqq L=n^{1 / 6-}$,

$$
e_{n}^{x}(y)=a_{n}\left[\sin \left(\lambda_{n}^{x}\right)^{1 / 2}\left(y+b_{n}\right)+O\left(n^{-1 / 2} L^{3}\right)\right]
$$

by an elementary appraisal, and from the inequality $1=\int\left(e_{n}^{x}\right)^{2} \geqq a_{n}^{2}[1 / 2-$ $o(1)] 2 L$, the evaluation $\lambda_{n}^{\infty}=2 n$, and the rapid vanishing of $\Delta q$, it develops that $e_{n}^{x}(y)=O\left(n^{-1 / 2+}\right)$ and

$$
\lambda_{n}^{x}=2 n+O\left(n^{-1 / 6+}\right) \quad(n \uparrow \infty) .
$$

The same trick applies to the eigenfunction:

so

$$
\frac{\partial e_{n}^{x}(y)}{\partial x}=-e_{n}^{x}(x) \Delta q(x) \sum_{k \neq n}\left(\lambda_{k}^{x}-\lambda_{n}^{x}\right)^{-1} e_{k}^{x}(x) e_{k}^{x}(y),
$$

$$
\left|e_{n}^{x}(y)-e_{n}^{\infty}(y)\right| \leqq \sum_{k \neq n}|k-n|^{-1} O\left(k^{-1 / 6+}\right) \times O\left(n^{-1 / 12+}\right)=O\left(n^{-1 / 4+}\right) .
$$

But the Hermite function $e_{n}^{\infty}(y)=O\left(n^{-1 / 4}\right)$ by routine appraisal, so $e_{n}^{x}(y)=$ $O\left(n^{-1 / 4+}\right)$, and this is improved to $O\left(n^{-1 / 4}\right)$ plain by substituting back; in particular, $e_{n}(y)=e_{n}^{-\infty}(y)=O\left(n^{-1 / 4}\right)$. The improvement also applies to the eigenvalue: $\lambda_{n}^{x}=2 n+O\left(n^{-1 / 2}\right)$, independently of $x$. 
Step 2 is to explain the plan of the proof: $Q$ and $Q^{0}$ have common spectrum $\lambda_{n}=2 n$ and if also $c_{n}^{+} / c_{n}^{-}=1$, then their eigenfunctions $e_{n}$ and $e_{n}^{0}$ are common multiplies of $w_{ \pm}$and $w_{ \pm}^{0}$, respectively, for $\lambda=2 n$. This permits you to write ${ }^{15}$

$$
\begin{gathered}
-\sum \frac{\left[\Delta e_{n}(x)\right]^{2}}{\lambda_{n}-\lambda_{-1}}+\frac{\Delta w_{-}(x, \lambda) \Delta w_{+}(x, \lambda)}{\Delta(\lambda)} \text { evaluated at } \lambda=\lambda_{-1} \\
=\frac{1}{2 \pi \sqrt{-1}} \int \frac{\Delta w_{-}(x, \lambda) \Delta w_{+}(x, \lambda)}{\left(\lambda-\lambda_{-1}\right) \Delta(\lambda)} d \lambda \equiv I
\end{gathered}
$$

for fixed $x$ and $\lambda_{-1}<0$, the sum being taken over $n \leqq N$ and the integral about a circle of odd radius $2 N+1$ enclosing $\lambda_{-1}$. The plan is to prove that $I=o(1)$ as $N \uparrow \infty$. Then

$$
\frac{\Delta w_{-}(x, \lambda) \Delta w_{+}(x, \lambda)}{\Delta(\lambda)} \text { at } \lambda=\lambda_{-1}=\sum \frac{\left[\Delta e_{n}(x)\right]^{2}}{\lambda_{n}-\lambda_{-1}} .
$$

The estimate $w=w^{0}\left[1+O\left(\lambda^{-1 / 2}\right)\right]$ for $\lambda \downarrow-\infty$ permits the left-hand side to be replaced by the free Green's function $\left(Q^{0}-\lambda_{-1}\right)_{x x}^{-1} \times O\left(1 / \lambda_{-1}\right)$, all of which is $o\left(1 / \lambda_{-1}\right)$ for $\lambda_{-1} \downarrow-\infty$, and comparison with the sum implies $\left[\Delta e_{n}(x)\right]^{2}=0$ for every $n \geqq 0 . Q=Q^{0}$ is immediate from that.

Step 3 is to carry out the appraisal of $I$. Let $\Delta^{x}(\lambda)$ be the value of $\left[w_{-}^{0}, w_{+}\right]$at $x$ and $G_{\lambda}^{x}$ the Green's function for the operator $Q^{x}$ based upon the potential $q^{x}(y)=$ $y^{2}-1$ or $q(y)$ according as $y<x$ or not. Then $G_{\lambda}^{x}(x, y)=-\left[\Delta^{x}(\lambda)\right]^{-1} w^{0}(x, \lambda)$ $w_{+}(y, \lambda)$ for $y>x$, and the integral $I$ of Step 2 is recognized as a sum of four pieces, of which

$$
I_{1}=\int\left[G_{\lambda}^{x}(x, x)-G_{\lambda}^{\infty}(x, x)\right] \frac{d \lambda}{\lambda-\lambda_{-1}}
$$

and

$$
I_{2}=\int G_{\lambda}^{x}(x, x)\left[\frac{\Delta^{x}(\lambda)}{\Delta^{\infty}(\lambda)}-1\right] \frac{d \lambda}{\lambda-\lambda_{-1}}
$$

are typical. The bracketed part of the first integrand is

$$
G_{\lambda}^{x}\left[q^{x}-q^{\infty}\right] G_{\lambda}^{\infty}(x, x)=\int_{x}^{\infty} G_{\lambda}^{x}(x, y) \Delta q(y) G_{\lambda}^{\infty}(y, x) d y,
$$

and so is controlled by

$$
\left[\sum \frac{\left[e_{n}^{x}(x)\right]^{2}}{\left|\lambda_{n}^{x}-\lambda\right|^{2}} \sum \frac{\left[e_{n}^{\infty}(x)\right]^{2}}{\left|\lambda_{n}^{\infty}-\lambda\right|^{2}}\right]^{1 / 2} \leqq \sum_{n \neq N} \frac{O\left(n^{-1 / 2}\right)}{|n-N|^{2}}=O\left(N^{-1 / 2}\right)
$$

by the preliminary estimates $e_{n}^{x}=O\left(n^{-1 / 4}\right)$ and $\lambda_{n}^{x}=2 n+O\left(n^{-1 / 2}\right)$ of Step 1 . This disposes of $I_{1}=O\left(N^{-1 / 2}\right)$. The discussion of $I_{2}$ is similar: The preliminary estimates 
provide the appraisals:

$$
\frac{\Delta^{x}(\lambda)}{\Delta^{\infty}(\lambda)}=\prod_{n=0}^{\infty} \frac{\lambda-\lambda_{n}^{x}}{\lambda-\lambda_{n}^{\infty}}=1+O\left(N^{-1 / 2+}\right)^{16}
$$

and

$$
\left|G_{\lambda}^{x}(x, x)\right| \leqq \sum_{n \neq N} \frac{O\left(n^{-1 / 2}\right)}{|n-N|}=O\left(N^{-1 / 2+}\right),
$$

so that $I_{2}=O\left(N^{-1+}\right)$. The proof is finished.

\section{Behaviour of Norming Constants}

The next item of business is to confirm that $c_{n}^{+} / c_{n}^{-}$tends rapidly to 1 as $n \uparrow \infty$. This is the most difficult part.

Step 1 . Let $f_{n}^{0}$ be any solution of $Q^{0} f=\lambda_{n} f$ with $\left[f_{n}^{0}, e_{n}^{0}\right]=1$. Then $D\left[f_{n}^{0}, e_{n}\right]=$ $e_{n} f_{n}^{0} \Delta q$, so $I_{n}=\int e_{n} f_{n}^{0} \Delta q=c_{n}^{+}-c_{n}^{-}$and, by the connection rule, it suffices to prove that this integral vanishes rapidly as $n \uparrow \infty$. Now you may take

$$
f_{n}^{0}(x)=n^{-1 / 2} 2^{n / 2}(n !)^{-1 / 2} e^{x^{2} / 2} \int_{0}^{\infty} \sin (2 x y) e^{-y^{2}} y^{n} d y,
$$

the upper [lower] trigonometrical function being employed for even [odd] $n$, and it is easy to see that

$$
f_{n}^{0}(x)=O\left(n^{-3 / 4}\right) e^{x^{2} / 2} ; \text { also, } e_{n}^{2}(x)=O\left[x^{2 n+c} e^{-x^{2} / 2}\right],
$$

independently of $|x| \geqq 1$ and $n$, if $c \geqq \Delta q \cdot{ }^{17}$ Let $L=n^{1 / 6-}$ as before. The estimates imply the rapid vanishing of the contribution to $I_{n}=\int e_{n} f_{n}^{0} \Delta q$ from $|x| \geqq L$. The contribution from $|x|<L$ is more subtle. To begin with, for $|x|<L, Q h=\lambda h$ has two independent solutions of the form

$$
\begin{aligned}
& h_{1}(x, \lambda)=A \cos \sqrt{\lambda}(x+L)+\lambda^{-1 / 2} B \sin \sqrt{\lambda}(x+L) \\
& h_{2}(x, \lambda)=A \sin \sqrt{\lambda}(x+L)-\lambda^{-1 / 2} B \cos \sqrt{\lambda}(x+L),
\end{aligned}
$$

$A$ and $B$ being formal power series in $\lambda^{-1}$ determined by $-2 \lambda A^{\prime}+B^{\prime \prime}=q B$, $2 B^{\prime}+A^{\prime \prime}=q A$, and the values $A=1, A^{\prime}=0, B=0, B^{\prime}=0$ at $x=-L$. The error involved in breaking off such a series is of the naively apparent order after multiplication by $\Delta q(x)$. Now for $|x|<L, e_{n}$ is a superposition of $h_{1}$ and $h_{2}$ evaluated at $\lambda=2 n$, of amplitude $n^{-1 / 4}$, while $f_{n}^{0}$ is a similar superposition of the corresponding functions $h^{0}$ for $Q^{0}$, of amplitude $n^{-3 / 4}$, so that, up to rapidly vanishing stuff, $I_{n}$ is a sum of integrals $\int h_{i} h_{j}^{0} \Delta q$ over $|x|<L$, with coefficients of magnitude $n^{-1}$ or

better. Terms involving $\sin \cos , \sin ^{2}-1 / 2$, or $\cos ^{2}-1 / 2$ are negligible as $n \uparrow \infty$

16 The possibility of an exponential factor $e^{a+b \lambda}$ is obviated by the fact that $\Delta^{x}=\Delta^{\infty}[1+o(1)]$ as $\lambda \downarrow \infty$.

$17 \sum e^{-2 n t} e_{n}^{2}(x)$ represents the elementary solution of $\partial p / \partial t=-Q p$ on diagonal which is overestimated by the product of $e^{t c}$ and the free elementary solution on diagonal. The latter is $\left[2 \pi\left(1-\varepsilon^{2}\right)\right]^{-1 / 2}$ $\exp \left[-x^{2}(1-\varepsilon)(1+\varepsilon)^{-1}\right]$ with $\varepsilon=e^{-2 t}$. Now take $\varepsilon=1 / 2 x^{2}$. 
in view of the fact that $\Delta q$ is of class $\mathbf{S}$, and the upshot is that $I_{n}$ is controlled, up to rapidly vanishing stuff, by

and

$$
\int_{-L}^{L}\left[A A^{0}+\lambda^{-1} B B^{0}\right] \Delta q=2\left(A^{0} B-A B^{0}\right)-\left[A, A^{0}\right]-\lambda^{-1}\left[B, B^{0}\right]
$$

$$
\int_{-L}^{L}\left[A B^{0}-A^{0} B\right] \Delta q=2 B B^{0}-\left[A, B^{0}\right]-\left[A^{0}, B\right]+2 \lambda\left(A A^{0}-1\right),
$$

the right-hand sides being evaluated at $\lambda=2 n$ and $x=L$. Now the key to the proof is the

Lemma. The formal power series $A-A^{0}$ and $B-B^{0}$ evaluated at $x=L$ vanish to all orders in $\lambda^{-1}$.

This will be proved in Steps 2 and 3 below. The rapid vanishing of $I_{n}$ is easily confirmed with its help. The first integral is plainly negligible, while the second reduces to

$$
C=2 B^{2}-2[A, B]+2 \lambda\left(A^{2}-1\right),
$$

evaluated at $x=L$. But $C$ is independent of $x\left[C^{\prime}=0\right]$ and vanishes at $x=-L$ so $C(L)=0$, too, completing the proof.

Step 2 is preparatory to the proof of the lemma. Let $H_{0}=\int q, H_{1}=\int\left[(1 / 2) q^{2}\right]$, $H_{2}=\int\left[(1 / 2) q^{3}+(1 / 4)\left(q^{\prime}\right)^{2}\right], \ldots, H_{n}=\int I_{n}[q]$, etc. be the usual KDV invariants. ${ }^{18}$ They have no meaning in the class $\mathbf{Q}$, but the relative invariants ${ }^{19}$

$$
J_{n}=\int\left[I_{n}-I_{n}^{0}\right]
$$

do, and since the $H$ 's represent spectral information when they make sense, it is not surprising that the $J$ 's vanish identically in $\mathbf{Q}$. The proof employs the fact that if $p(t, x, y)$ is the elementary solution of $\partial p / \partial t=-Q p$, then $\Sigma e^{-\lambda_{n} t}=\int p(t, x, x)$, so that $\int\left[p(t, x, x)-p^{0}(t, x, x)\right]$ vanishes identically. But this relative trace admits the development ${ }^{20}(4 \pi t)^{-1 / 2}\left[J_{0} t+J_{1} t^{2}+\right.$ etc. $]$ for $t \downarrow 0$. The vanishing of $J_{n}(n \geqq 0)$ is now plain.

Step 3 is the proof of the lemma. Introduce the discriminant of $Q$ in the interval $|x|<L: D(\lambda)=h_{1}+\lambda^{-1 / 2} h_{2}^{\prime}$ evaluated at $x=L$. The logarithm of $D(\lambda)$ admits a development ${ }^{21}$ in powers $1 / 2-n(n \geqq 0)$ of $\lambda \downarrow-\infty$, in which the coefficients may be expressed as short KDV invariants $\int_{-L}^{L} I_{n}$ plus gradients of long KDV invariants $H_{j}$ evaluated at $x= \pm L$; similarly, the logarithm of $D(\lambda) / D^{0}(\lambda)$ admits a development with (vanishing) relative invariants in place of short invariants, plus rapidly vanishing contributions from $x= \pm L$. The upshot is that $\ell \mathrm{g}\left[D(\lambda) / D^{0}(\lambda)\right]$ vanishes rapidly as $\lambda \downarrow-\infty$. The proof of the lemma is finished by comparison with the development

$$
D(\lambda)=e^{2 L \sqrt{-\lambda}} \times\left[A-(2 \lambda)^{-1} B+(-\lambda)^{-1 / 2}\left(B+A^{\prime} / 2\right)\right] \text { evaluated at } x=L
$$

18 McKean-Moerbeke [1975], for instance.

$19 I_{n}^{0}=I_{n}\left[x^{2}-1\right]$.

20 McKean-Moerbeke [1975] can serve as a model for the proof.

21 McKean-Moerbeke [1975]. 
and its counterpart for $D^{0}(\lambda)$.

Proof of the surjection. Choose a potential $q$ of class $\mathbf{Q}$. The numbers $t_{n}=\ell \mathrm{g} c_{n}^{+} / c_{n}^{-}$ vanish rapidly as $n \uparrow \infty$ and so may be used to form the tangent vector $\mathbf{X}=\Sigma t_{n} \mathbf{X}_{n}$. The flow $e^{-t \mathbf{x}}: 0<t \leqq 1$ moves $q$ to some point $q^{0} \in \mathbf{Q}$, reducing all the numbers $c_{n}^{+} / c_{n}^{-}$to unity. But $q^{0}$ can only be the origin $q^{0}(x)=x^{2}-1$, by uniqueness, and surjectivity follows by reversing the flow: $q=e^{\mathbf{x}} q^{0}$. The proof is finished.

\section{Canonically Conjugate Variables}

The region of definition of the parameters $t_{n}(n \geqq 0)$ is extended from $\mathbf{Q}$ into the ambient space by the rule

$$
t_{n}=\lim _{x \uparrow \infty} \ell \mathrm{g}\left[(-1)^{n} e_{n}(x) / e_{n}(-x)\right] .
$$

It is to be proved that the variables $2 \lambda_{i}$ and $t_{j}$ are canonically conjugate relative to the bracket $[F, G]=\int \nabla F D \nabla G$. The vanishing of $\left[\lambda_{i}, \lambda_{j}\right]$ was already noted; also $\left[t_{i}, 2 \lambda_{j}\right]=1$ or 0 according to whether $i=j$ or not since the flow $\partial q / \partial t=\mathbf{X}_{j} q=$ $\left[q, 2 \lambda_{j}\right]$ advances $t_{i}$ at speed 1 if $i=j$ and not at all if $i \neq j$. The only point still at issue is the vanishing of $\left[t_{i}, t_{j}\right]$.

Proof. The gradient of $t_{n}$ is found to be $\mathrm{b}^{22} \nabla t_{n}=\left(\Delta^{*}\right)^{-1}\left[w_{+}^{*} w_{-}-w_{-}^{*} w_{+}\right]$evaluated at $\lambda=\lambda_{n}$. This expression is of the form $e_{n} f_{n}$ with $Q f_{n}=\lambda_{n} f_{n}$ since $e_{n}$ is proportional to $e^{-t_{n} / 2} w_{-}=e^{t_{n} / 2} w_{+}$, while $h=e^{t_{n} / 2} w_{+}-e^{-t_{n} / 2} w_{-}$satisfies $Q h^{\circ}=$ $\lambda h^{\circ}+h$ and $h=0$ for $\lambda=\lambda_{n}$; also, $\left[f_{n}, e_{n}\right]=1$ is easily proved; finally, $\nabla t_{n}=O\left(x^{-1}\right)$ at $\pm \infty$ since $e_{n}(x)$ behaves like $x^{n} e^{-x^{2} / 2}$ and $f_{n}(x)$ like $x^{-n-1} e^{x^{2} / 2}$. Now it is plain that $\left[t_{i}, t_{j}\right]$ exists. It vanishes automatically if $i=j$, while if $i \neq j$, the same follows from the identity $K h=2 \lambda D h$ for $h=e_{n} f_{n}, \lambda=\lambda_{n}$, and $K=q D+D q-(1 / 2) D^{3}$ : in detail, $2 \lambda_{j}\left[t_{i}, t_{j}\right]=2 \lambda_{j} \int h_{i} D h_{j}=\int h_{i} K h_{j}=-\int h_{j} K h_{i}=-2 \lambda_{i} \int h_{j} D h_{i}=2 \lambda_{i} \int h_{i} D h_{j}=$ $2 \lambda_{i}\left[t_{i}, t_{j}\right]$. The only step needing clarification is that from $\int h_{i} K h_{j}$ to $-\int h_{j} K h_{i}$. This is dealt with by noting $1=\left[f_{n}, e_{n}\right] \sim-2 x e_{n} f_{n}$, so that $2 q h_{i} h_{j} \sim 2 x^{2} h_{i} h_{j} \sim 1 / 2$ and the contributions from $\pm^{\infty} \infty$ in the necessary partial integration cancel out.

Amplification 1. The formula $\nabla t_{n}=e_{n} f_{n}$ leads to a direct evaluation of $\left[t_{i}, 2 \lambda_{j}\right]$ : if $i \neq j$, the bracket is proportional to

$$
2 \lambda_{j} \int e_{i} f_{i} D e_{j}^{2}=\int e_{i} f_{i} K e_{j}^{2}=-\int e_{j}^{2} K e_{i} f_{i}=2 \lambda_{i} \int e_{i} f_{i} D e_{j}^{2}
$$

and so vanishes, while if $i=j$, it is

$$
2 \int e_{i} f_{i} D e_{i}^{2}=-2 \int e_{i}^{2} D e_{i} f_{i}=\int e_{i}^{2}\left[f_{i}, e_{i}\right]=\int e_{i}^{2}=1 .
$$

\section{A More Global View}

Now the spectrum $\lambda_{n}(n \geqq 0)$ and the logarithmic norming constants $t_{n}(n \geqq 0)$ should provide a complete set of canonically conjugate coordinates not just

$22 w_{-}=e^{t_{n}} w_{+}$at $\lambda=\lambda_{n}$. The gradient of this relation is now taken and combined with the following facts: a) $\partial w_{ \pm}(x) / \partial q(y)$ vanishes at $x=y$, b) $\nabla \lambda_{n}=e_{n}^{2}$, c) $e_{n}=c e^{-t_{n} / 2} w_{-}=c e^{t_{n} / 2} w_{+}$, d) $c^{2}=-1 / \Delta^{\cdot}\left(\lambda_{n}\right)$. The spot signifies differentiation with regard to $\lambda$. 
in $\mathbf{Q}=\mathbf{Q}\left[x^{2}-1\right]$ but in an appropriate ambient space, $x^{2}-1+\mathbf{S}$, say, or wider, i.e., the general spectral class $\mathbf{Q}$ obtained by fixing $\lambda_{n}(n \geqq 0)$ not at $\lambda_{n}\left[x^{2}-1\right]=2 n$, but at some other values with realistic comportment as $n \uparrow \infty$, should meet the class $\mathbf{P}$ obtained by a similar fixing of $t_{n}(n \geqq 0)$ in a single point; moreover, these Q's and P's should be the leaves of transversal foliations of the ambient space. The purpose of the present section is to examine the geometry of this picture in the special leaf $\mathbf{Q}=\mathbf{Q}\left[x^{2}-1\right]$. The situation is outlined in the next three articles.

\section{Completeness}

The directions $\nabla \lambda_{i}=e_{i}^{2}$ are normal to the leaf $\mathbf{Q}$ at $q$, while the directions $\partial q / \partial t_{j}=$ $D e_{j}^{2}$ are tangent to it, as expressed by the first canonical identity $\left[\lambda_{i}, \lambda_{j}\right]=0$, and it is a source of satisfaction that these directions together span $\mathbf{L}^{2}$ so that no direction is left unclassified. This is termed completeness. The same holds for the leaf $\mathbf{P}$ at $q: \nabla t_{i}=e_{i} f_{i}$ is normal, $\partial q / \partial \lambda_{j}=D e_{j} f_{j}$ is tangent in view of the third canonical identity $\left[t_{i}, t_{j}\right]=0$, and the same type of completeness obtains. Contrariwise, $\nabla \lambda_{i}$ and $D \nabla t_{j}\left[D \nabla \lambda_{i}\right.$ and $\left.\nabla t_{j}\right]$ need not span: for example, at the origin $q^{0}(x)=x^{2}-1$, they span only the even [odd] functions.

\section{Transversality}

The second canonical identity

$$
\left[t_{i}, 2 \lambda_{j}\right]=2 \int e_{i} f_{i} D e_{j}^{2}=1(i=j)=0(i \neq j)
$$

implies that the normal spaces to $\mathbf{Q}$ and $\mathbf{P}$ meet only in the null function in view of what went before, and it is a further source of satisfaction to find that these two spaces together span $\mathbf{L}^{2}$, expressing a transversality of $\mathbf{Q}$ and $\mathbf{P}$; the same holds for the tangent spaces, confirming the picture of Fig. 2.

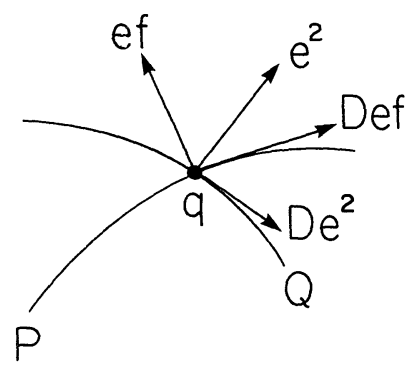

Fig. 2.

\section{Independence}

The second canonical identity also implies a stringent independence of normal and tangent directions. For example, if the normal directions $\nabla \lambda_{n}=e_{n}^{2}$ are divided into two classes, then nothing in the span of the first class lies in the span of the second, the point being that anything in the intersection is simultaneously perpendicular to 
$D e_{i}^{2}$ and to $D e_{i} f_{i}$ and is null, by completeness and transversality. This type of independence indicates that $\mathbf{Q}$ is a smooth submanifold of the ambient space; naturally, the same holds for $\mathbf{P}$.

Proof. The rest of this section is occupied by the proof of completeness and transversality.

Step 1. Fix a complex number $\lambda$ outside the spectrum, let $G=G_{\lambda}(x, y)$ be the Green function $(Q-\lambda)_{x y}^{-1}$, and introduce the operator

$$
H: f \rightarrow \int( \pm 1) \times\left[G^{2}(x, y)-G(x, x) G(y, y)\right] f(y) d y,
$$

the + sign being employed if $y>x$ and the - sign otherwise. The present step contains appraisals of $H$ required below. $G(x, x) \leqq c_{1}\left(x^{2}-\lambda\right)^{-1 / 2}$ for $\lambda<0$ by routine estimation ${ }^{23}$. This provides the preliminary bound:

$$
\begin{aligned}
& \left|G^{2}(x, y)-G(x, x) G(y, y)\right| \\
& \quad \leqq 2 \Sigma\left|\lambda_{n}-\lambda\right|^{-1} e_{n}^{2}(x) \Sigma\left|\lambda_{n}-\lambda\right|^{-1} e_{n}^{2}(y) \\
& \leqq 2 \max _{n \geqq 0}\left|\frac{\lambda_{n}+1}{\lambda_{n}-\lambda}\right|^{2} G_{-1}(x, x) G_{-1}(y, y) \\
& \leqq c_{2}(\lambda)\left[\left(x^{2}+1\right)\left(y^{2}+1\right)\right]^{-1 / 2},
\end{aligned}
$$

which implies

$$
\|H f\|_{2}^{2} \leqq c_{2}^{2} \int \frac{d x}{x^{2}+1} \int \frac{d y}{y^{2}+1}\|f\|_{2}^{2}=c_{2}^{2} \pi^{2}\|f\|_{2}^{2} .
$$

Thus, $H$ is a bounded operator on $\mathbf{L}^{2}$ : in particular, $c_{2}(\lambda)=O\left(N^{2}\right)$ on circles of odd radius $2 N+1$, so that $\|H\|_{2}=O\left(N^{2}\right)$ there. Better information is available for $\lambda<0:\|H\|_{2}=O\left(\lambda^{-1}\right)$. Now $H$ is inverted by $K-2 \lambda D$ with $K=q D+D q-$ $(1 / 2) D^{3}$, as a routine computation shows. This will lead to the bound $\|D H\|_{2}=O\left(N^{3}\right)$ on odd circles. To begin with, $(K-2 \lambda D) H=1$ implies $D H f=(L-2 \lambda)^{-1}\left(1-q^{\prime} H\right)$ $f+g$ with $L=-(1 / 2) D^{2}+2 q$ and $L g=2 \lambda g$. Next, it is necessary to observe that the distant spectrum of $L$ approximates that of $-(1 / 2) D^{2}+2\left(x^{2}-1\right)$, namely $4 n(n \geqq 0)$. It follows that $g=0$ : indeed, $q^{\prime} H f \in \mathbf{L}^{\infty}$ by the preliminary bound and $H f \in \mathbf{L}^{2}$, while any non-vanishing solution of $L g=2 \lambda g$ is exponentially large if $2 \lambda$ is not in the spectrum of $L$ and could only unbalance the identity for $D H f$. The upshot is that $D H=(L-2 \lambda)^{-1}\left(1-q^{\prime} H\right)$ plain. Now $(L-2 \lambda)^{-1}$ and $G$ have a similar behaviour for $|\lambda|=2 N+1$, so the idea of the proof will be adequately conveyed by confirming $\left\|G\left(x^{2}+1\right)^{1 / 2} H\right\|_{2}=O\left(N^{3}\right)$ on odd circles. The preliminary bound implies $\left\|\left(x^{2}+1\right)^{1 / 2} H f\right\|_{\infty}=O\left(N^{2}\right)\|f\|_{2}$, so it suffices to overestimate $\|G h\|_{2}$ by $O(N)\|h\|_{\infty}$ for $h \in \mathbf{L}^{2} \cap \mathbf{L}^{\infty}$. That is easy:

$$
\begin{aligned}
\|G h\|_{2}^{2} & =\Sigma\left|\lambda_{n}-\lambda\right|^{-2}\left(e_{n}, h\right)^{2} \leqq c_{2}(2 N+1) \Sigma\left|\lambda_{n}+1\right|^{-2}\left(e_{n}, h\right)^{2} \\
& =O\left(N^{2}\right)\left\|G_{-1} h\right\|_{2}^{2}=O\left(N^{2}\right)\left\|G_{-1}^{0} 1\right\|_{2}^{2}\|h\|_{\infty}^{2},
\end{aligned}
$$

$G_{-1}^{0}$ being the free Green's function for $q^{0}(x)=x^{2}-1$ and $\lambda=-1$. But $f=G_{-1}^{0} 1$

$23 G(x, x) \leqq \int_{0}^{\infty} e^{\lambda t} e^{c t}\left[2 \pi\left(1-\varepsilon^{2}\right)\right]^{-1 / 2} \exp \left[-x^{2}(1-\varepsilon)(1+\varepsilon)^{-1}\right] d t$ with $\lambda<0, c \geqq \Delta q$, and $\varepsilon=e^{-2 t}$. 
looks like $x^{-2}$ far out since it solves $\left(Q^{0}+1\right) f=1$ and vanishes at $\pm \infty$. The proof is finished.

Step 2. $e_{i}^{2}$ and $e_{j} f_{j} \operatorname{span} \mathbf{L}^{2}$. The residue of $H$ at $\lambda=2 n$ is $R_{n}=e_{n}^{2} \otimes e_{n} f_{n}-e_{n} f_{n} \otimes$ $e_{n}^{2}$, and the fact that $\|H\|_{2}=O\left(N^{2}\right)$ on odd circles justifies the expansion $H_{3}=\partial^{3} H / \partial \lambda^{3}=6 \Sigma\left(\lambda_{n}-\lambda\right)^{-4} R_{n}$ off spectrum, by a self-evident application of the Cauchy integral for $H_{3}$. Now let $f \in \mathbf{L}^{2}$ be perpendicular to $e_{i}^{2}$ and $e_{j} f_{j}$. Then $H_{3} f=0$ and the vanishing of $\|H\|_{2}$ at $\lambda=-\infty$ implies that $H f=0$, too, so that $f=(K-2 \lambda D) H f=0$, as well.

Step 3. De $e_{i}^{2}$ and $D e_{j} f_{j}$ span $\mathbf{L}^{2}$. The proof is similar. The extra technicalities required will be found in Step 4.

Step 4. $e_{i}^{2}$ and $D e_{j}^{2}$ span $\mathbf{L}^{2}$. The fact that $\|D H\|_{2}=O\left(N^{3}\right)$ on odd circles justifies the expansion $D H_{4}=D \partial^{4} H / \partial \lambda^{4}=24 \Sigma\left(\lambda_{n}-\lambda\right)^{-5} D R_{n}$. Let $f \in \mathbf{L}^{2}$ be perpendicular to $e_{n}^{2}(n \geqq 0)$. Then $R_{n} f$ is a multiple of $e_{n}^{2}$ and $D H_{4} f$ belongs to the span of $D e_{n}^{2}(n \geqq 0)$. The same follows for $D H f$, and the fact that $\|2 \lambda D H f+f\|=$ $o(1)$ as $\lambda \downarrow-\infty$, finishes the proof. This final point requires justification; it makes use of the identity $D H=(L-2 \lambda)^{-1}\left(1-q^{\prime} H\right)$ of Step 1. $\left\|2 \lambda(L-2 \lambda)^{-1} f+f\right\|_{2}$ $=o(1)$ is plain, so it suffices to prove $\left\|(L-2 \lambda)^{-1} q^{\prime} H f\right\|_{2}=o\left(\lambda^{-1}\right)$; as in Step 1, the idea will be adequately conveyed by proving $\left\|G\left(x^{2}+1\right)^{1 / 2} H f\right\|_{2}=o\left(\lambda^{-1}\right)$. The bound $G(x, x) \leqq c_{1}\left(x^{2}-\lambda\right)^{-1}$ for $\lambda<0$ implies $|H f| \leqq c_{1}^{2}\left(x^{2}-\lambda\right)^{-1 / 2}$ $\int\left(y^{2}-\lambda\right)^{-1 / 2}|f| d y=o\left[\lambda^{-1 / 4}\left(x^{2}-\lambda\right)^{-1 / 2}\right]$, so

$$
\begin{aligned}
\left\|G\left(x^{2}+1\right)^{1 / 2} H f\right\|_{2}^{2} & =o\left(\lambda^{-1 / 2}\right)\left\|G\left(x^{2}+1\right)^{1 / 2}\left(x^{2}-\lambda\right)^{-1 / 2}\right\|_{2}^{2} \\
& =o\left(\lambda^{-1 / 2}\right)\left\|G\left(x^{2}+1\right) G\left(x^{2}-\lambda\right)^{-1}\right\|_{1} .
\end{aligned}
$$

But $\left\|G\left(x^{2}+1\right)\right\|_{\infty}=O(1)$, as may be confirmed directly for $G^{0}$ and carries over to $G$ itself, while

$$
\left\|G\left(x^{2}-\lambda\right)^{-1}\right\|_{1}=\int d x \int G(x, y)\left(y^{2}-\lambda\right)^{-1} d y=O\left(\lambda^{-1}\right) \int\left(y^{2}-\lambda\right)^{-1} d \lambda=O\left(\lambda^{-3 / 2}\right)
$$

by exchange of integrals. The upshot is the necessary bound $\left\|G\left(x^{2}+1\right)^{1 / 2} H f\right\|_{2}=$ $o\left(\lambda^{-1}\right)$ with nothing much to spare.

Step 5. $e_{i} f_{i}$ and $D e_{j} f_{j} \operatorname{span} \mathbf{L}^{2}$. The proof is similar. The discussion is finished.

\section{Transversal Flows}

The present section contains a brief discussion of the transversal flows $\partial q / \partial t=\mathbf{Y}_{n} q$ produced by the vector fields $\mathbf{Y}_{n}: q \rightarrow D \nabla t_{n}:$ it is desired to move the $n^{\text {th }}$ eigenvalue $\lambda_{n}$ at speed 1 keeping the rest of the spectrum and all of the norming constants fixed, and there is a simple recipe for that ${ }^{24}$.

\section{Individual Flows}

Let $n=0$ and fix $q^{0}$. The ground state $e_{0}^{0}$ is positive, so $q^{-}=q^{0}-2 D^{2} \ell \mathrm{g} e_{0}^{0}$ makes sense, and it turns out that the corresponding operator $Q^{-}$has a) the same spectrum

24 Deift-Trubowitz [1979] may be consulted for details omitted here. 
as $Q^{0}$ but with $\lambda_{0}^{0}$ excised, b) eigenfunctions $e_{n}^{-}=\left(\lambda_{n}^{0}-\lambda_{0}^{0}\right)^{-1 / 2}\left(e_{0}^{0}\right)^{-1}\left[e_{0}^{0}, e_{n}^{0}\right]$ $(n \geqq 1)$, and c) the same norming constants, i.e., $e_{n}^{-}(x) / e_{n}^{-}(-x) \sim e_{n}^{0}(x) / e_{n}^{0}(-x)$ as $x \uparrow \infty$ for $n \geqq 1$. The eigenvalue $\lambda_{0}^{0}$ is now restored to a different place $\lambda_{0}^{+}<\lambda_{1}^{0}$ by a similar recipe: $Q^{-}$has an eigenfunction $f_{0}^{-}$with eigenvalue $\lambda_{0}^{+}$which is of one signature $(+)$ and satisfies $f_{0}^{-}(x) / f_{0}^{-}(-x) \sim e^{t_{0}}$ as $x \uparrow \infty$, and it turns out that the new operator $Q^{+}$with potential $q^{+}=q^{-}-2 D^{2} \ell \mathrm{g} f_{0}^{-}$has a) spectrum $\lambda_{0}^{+}<$ $\lambda_{1}^{0}<\lambda_{2}^{0}<$ etc., b) eigenfunctions

$$
e_{0}^{+}=\left(f_{0}^{-}\right)^{-1}\left[\int\left(f_{0}^{-}\right)^{-2}\right]^{-1 / 2}, e_{n}^{+}=\left(\lambda_{n}^{0}-\lambda_{0}^{+}\right)^{-1 / 2}\left(f_{0}^{-}\right)^{-1}\left[f_{0}^{-}, e_{n}^{-}\right](n \geqq 1),
$$

and c) the same norming constants $t_{n}^{+}=t_{n}^{0}$ as $Q^{0}$; moreover, it is easy to check that the map $q^{0} \rightarrow q^{+}$effects the flow $\partial q / \partial t=D \nabla t_{0}$ with parameter $t=\lambda_{0}^{+}-\lambda_{0}^{0}$. The auxiliary function $f_{0}^{-}$is easily computed from the eigenfunctions of $Q^{0}$ : it is proportional to $\left(e_{0}^{0}\right)^{-1}\left[e_{0}^{0}, f_{0}^{0}\right]$ with

$$
f_{0}^{0}=\left(\lambda_{0}^{+}-\lambda_{0}^{0}\right)^{-1} \times\left[e^{t_{0} / 2} w_{+}^{0}-e^{-t_{0} / 2} w_{-}^{0} \text { evaluated at } \lambda=\lambda_{0}^{+}\right] .
$$

The flow now takes the simple form $\Delta q=-2 D^{2} \ell \mathrm{g}\left[e_{0}^{0}, f_{0}^{0}\right]$.

\section{Multiple Flows}

The recipe is easily extended to multiple flows: several eigenvalues $\lambda_{i}^{0}(i \leqq n)$ are excised in their natural order by successive applications of the map $q^{0} \rightarrow q^{-}$and put back in the opposite order at new places $\lambda_{0}^{+}<\lambda_{1}^{+} \ldots<\lambda_{n}^{+}$to the left of $\lambda_{n+1}^{0}$ by use of the $\operatorname{map} q^{-} \rightarrow q^{+}$. The result may be put in the simple form:

$$
\Delta q=-2 D^{2} \ell \mathrm{g}\left[e_{0}^{0}, \ldots e_{n}^{0}, f_{0}^{0}, \ldots, f_{n}^{0}\right],
$$

with

$$
f_{i}^{0}=\left(\lambda_{i}^{+}-\lambda_{0}^{0}\right)^{-1} \times\left[e^{t_{i} / 2} w_{+}^{0}-e^{-t_{l} / 2} w_{-}^{0} \text { evaluated at } \lambda=\lambda_{i}^{+}\right],
$$

$\left[e_{0}^{0}, \ldots, f_{n}^{0}\right]$ being the Wronskian determinant. It is a pleasant feature of the recipe that if $\lambda_{i}^{+}=\lambda_{i}^{0}$ for $i \neq j$, then also $\left[e_{i}^{0}, f_{i}^{0}\right]=1$ for $i \neq j$, so that the big Wronskian simplifies to $\left[e_{j}^{0}, f_{j}^{0}\right]$, and the reduced formula $\Delta q=-2 D^{2} \ell \mathrm{g}\left[e_{j}^{0}, f_{j}^{0}\right]$ expresses the $j^{\text {th }}$ individual flow in the same manner as for $j=0$. The discussion of the case $n=\infty$ is not so simple and will not be entered upon.

\section{Charge}

To be candid, the matter is not quite so simple, even for $n<\infty$ : starting from the origin, the flow produces in $q(x)$ novel terms $c_{2}( \pm \infty) x^{-2}+c_{3}( \pm \infty) x^{-3}+$ etc. near $\pm \infty$ and so leads out of the familiar ambient space $x^{2}-1+\mathbf{S}$ : for example, $c_{2}( \pm \infty)= \pm(1 / 2) \Sigma\left[\lambda_{i}^{+}-\lambda_{i}^{0}\right]$ for any $q^{0} \in x^{2}-1+\mathbf{S}$. This only means that the ambient space must be enlarged to accommodate such behaviour; it seems reasonable to admit terms $c_{0}( \pm \infty)+c_{1}( \pm \infty) x^{-1}$ as well. The number $c_{0}(+\infty)-c_{0}(-\infty)$ is called the charge of $q$. The spectral interpretation of $c_{2}$ should have a counterpart for $c_{0}$ since it cannot be moved by any isospectral flow in view of the rapid vanishing of $D e_{n}^{2}$ at $\pm \infty$. The subject merits further investigation. 


\section{Example}

The proposed enlargement of the ambient space produces a wider spectral class $\mathbf{Q}\left[x^{2}-1\right]$. To see this requires an example. Let $q^{0}(x)=x^{2}-1$ and, in $q(x)=e^{t \mathbf{x}} q^{0}$ $=x^{2}-1-2 D^{2} \ell \mathrm{g}\left[1+\left(e^{t}-1\right) \int_{x}^{\infty}\left(e_{0}^{0}\right)^{2}\right]$, make $t \uparrow+\infty$ or $\downarrow-\infty$. The corresponding limits $q^{+}(x)$ and $q^{-}(x)=q^{+}(-x)$ are

$$
\begin{aligned}
& q^{+}(x)=x^{2}-1-2 D^{2} \int_{x}^{\infty}\left(e_{0}^{0}\right)^{2}=x^{2}-1+o(1) \text { at }-\infty=x^{2}+4+o(1) \text { at }+\infty \\
& q^{-}(x)=x^{2}-1-2 D^{2} \int_{\infty}^{x}\left(e_{0}^{0}\right)^{2}=x^{2}+3+0(1) \text { at }-\infty=x^{2}-1+o(1) \text { at }+\infty .
\end{aligned}
$$

The behaviour at $+\infty$ shows that $q^{+}$and $q^{-}$have escaped from $x^{2}-1+\mathbf{S}$; more drastically, the eigenfunction

$$
e_{0}(x)=e^{t / 2} e_{0}^{0}(x)\left[1+\left(e^{t}-1\right) \int_{x}^{\infty}\left(e_{0}^{0}\right)^{2}\right]^{-1}
$$

tends to 0 as $t \uparrow \infty$ or $\downarrow-\infty$. The explanation is that the former ground state 0 is not in the spectrum of $Q^{-}$or $Q^{+}$, the remaining eigenfunctions

$$
\begin{aligned}
& e_{n}^{+}=e_{n}^{0}-e_{0}^{0} \int_{x}^{\infty} e_{0}^{0} e_{n}^{0} \times\left[\int_{x}^{\infty}\left(e_{0}^{0}\right)^{2}\right]^{-1} \\
& e_{n}^{-}=e_{n}^{0}-e_{0}^{0} \int_{-\infty}^{x} e_{0}^{0} e_{n}^{0} \times\left[\int_{-\infty}^{x}\left(e_{0}^{0}\right)^{2}\right]^{-1}
\end{aligned}
$$

of index $n \geqq 1$ already forming a full set.

Proof. Let $f \in \mathbf{L}^{2}$ be perpendicular to $e_{n}^{+}(n \geqq 1)$, say. Then

$$
\int f e_{n}^{0}=\int f e_{0}^{0} \frac{\int_{x}^{\infty} e_{0}^{0} e_{n}^{0}}{\int_{x}^{\infty}\left(e_{0}^{0}\right)^{2}}=\int e_{0}^{0} e_{n}^{0} \int_{-\infty}^{x}\left[f e_{0}^{0} / \int^{\infty}\left(e_{0}^{0}\right)^{2}\right]
$$

for $n \geqq 0$, inclusive, so that

$$
f / e_{0}^{0}=\int_{-\infty}^{x}\left[f e_{0}^{0} / \int^{\infty}\left(e_{0}^{0}\right)^{2}\right]
$$

This leads to the contradictory evaluation of $f$ as a multiple of $\left(e_{0}^{0}\right)^{2}\left[\int_{x}^{\infty}\left(e_{0}^{0}\right)^{2}\right]^{-1}$ which is not in $\mathbf{L}^{2}$, provided $h=e_{0}^{0} \int_{-\infty}^{x}\left[f e_{0}^{0} / \int^{\infty}\left(e_{0}^{0}\right)^{2}\right]$ belongs to $\mathbf{L}^{2}$. The latter point requires a speck of ingenuity.

The $\operatorname{map} f \rightarrow h$ is dual to the map

$$
f \rightarrow \frac{e_{0}^{0}(x) \int_{x}^{\infty} f e_{0}^{0}}{\int_{x}^{\infty}\left(e_{0}^{0}\right)^{2}}
$$


and the latter is bounded: indeed, with $e$ in place of $e_{0}^{0}$ for simplicity,

$$
\begin{aligned}
\left\|f-e \int_{x}^{\infty} f e\right\|^{2} & =\int f^{2}+\int\left(\int_{x}^{\infty} e^{2}\right)^{2} d\left(\int_{x}^{\infty} f e\right)^{2}+\int e^{2} \frac{\left(\int_{x}^{\infty} f e\right)^{2}}{\left(\int_{x}^{\infty} e\right)^{2}} \\
& =\int f^{2}-\left.\frac{\left(\int_{x}^{\infty} f e\right)^{2}}{\int_{x}^{\infty} e^{2}}\right|_{-\infty} ^{\infty}=\int f^{2}-(e, f)^{2} \leqq\|f\|^{2},
\end{aligned}
$$

if $f$ vanishes near $+\infty$, and so also in general.

The eigenvalue $\lambda_{0}^{0}$ may now be restored to its original position by the recipe used for the individual flow of index $0: \lambda_{0}^{0}$ lies below the spectrum of $Q^{+}$, so that the latter has a positive eigenfunction $f_{0}^{+}$, and the new operator $Q$ with potential $q=q^{+}-2 D^{2} \ell \mathrm{g} f_{0}^{+}$has spectrum $\lambda_{n}=\lambda_{n}^{0}(n \geqq 0)$, i.e., it belongs to the spectral class $\mathbf{Q}\left[x^{2}-1\right]$. But this is not the old but some enlarged class: $\ell \mathrm{g} f_{0}^{+} \sim x^{2} / 2$ at $\pm \infty$, so

$$
\begin{aligned}
q(x) & =x^{2}-1+4-2+o(1)=x^{2}-1+2+o(1) \text { at }+\infty \\
& =x^{2}-1+0-2+o(1)=x^{2}-1-2+o(1) \text { at }-\infty ;
\end{aligned}
$$

in particular, $q$ has charge 4 .

\section{Theta Sums}

The so-called theta $\operatorname{sum} \theta_{x}\left(t_{0}, t_{1}, t_{2}\right.$, etc.) played a central role. The name is justified by recollecting ${ }^{25}$ what happens to the classical Riemann theta function of a nonsingular-elliptic curve

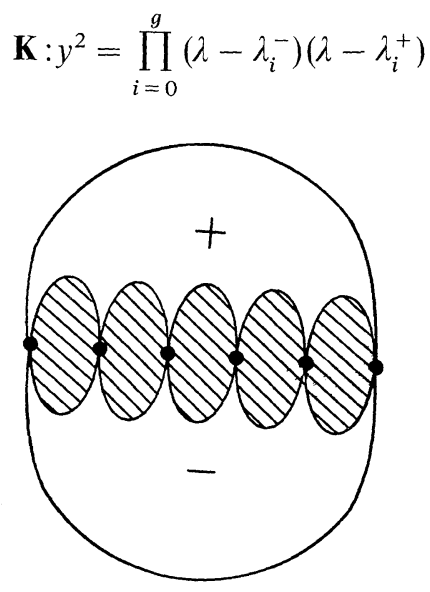

Fig. 3.

25 McKean [1979] gives details. 
of genus $g$ as the intervals $\left[\lambda_{i}^{-}, \lambda_{i}^{+}\right]$are pinched to single points $\lambda_{i}(i=0, \ldots, g)$. The curve becomes singular as in Fig. $3[g=5]$, falling apart into two sheets labelled plus and minus; simultaneously, the quadratic form figuring in the theta sum becomes huge, and the principal part of the theta function reduces to one of a number of finite sums $\theta_{k}$ indexed by $k=0, \ldots, g$, corresponding to a breaking up of the Jacobi variety $\mathbf{J}$ of $\mathbf{K}$ into $g+1$ connected pieces; this is caused by the breaking up of the space of divisors ${ }^{26} \mathfrak{p}_{1}, \ldots, \mathfrak{p}_{g}$ of degree $g$ into $g+1$ pieces according to the number $0 \leqq k \leqq g$ of points on the upper sheet ${ }^{27}$. The principal parts look like

$$
\theta_{k}(x)=\sum_{\substack{n_{i}=0 \text { or } 1(1 \leqq i \leqq g) \\ n_{1}+\ldots+n_{g}=k,}} e^{(x-c) \cdot n} e^{\sum_{i<j} n_{\imath} n_{j} \ell g\left|\lambda_{l}-\lambda_{J}\right|},
$$

in which the constant $c$ depends upon $k$ and $\mathfrak{x} \in \mathbf{J}$ is expressed by means of integrals of differentials of the first kind [DFK] summed over the typical divisor having $k$ points on the upper sheet, the sums being construed modulo periods. The forms $\omega_{j}=\left[\left(\lambda-\lambda_{j}\right)^{-1}-\left(\lambda-\lambda_{0}\right)^{-1}\right] d \lambda(j=1, \ldots, g)$ serve as a basis of DFK ${ }^{28}$ and

$$
\mathfrak{x}_{j}=\sum_{i=1}^{g} \operatorname{sign} \mathfrak{p}_{i} \times \int_{\infty}^{\mathfrak{p}_{l}} \omega_{j} \quad(j=1, \ldots, g),
$$

in which sign $\mathfrak{p}$ is $+1[-1]$ on the upper [lower] sheet and, in the $i^{\text {th }}$ integral, $\infty$ is taken on the same sheet as $\mathfrak{p}_{i}$. The same type of geometrical interpretation is available for the present theta sums: There is a singular curve

$$
\mathbf{K}: y^{2}=[\Delta(\lambda)]^{2}
$$

of infinite genus, some kind of Jacobi variety $\mathbf{J}$ broken up into uncountably many pieces indexed by the real number $x$, and a system of theta sums $\theta_{x}$, one to each piece. But what is the divisor $\mathfrak{p}_{0}, \mathfrak{p}_{1}, \mathfrak{p}_{2}, \ldots$ producing the argument $t_{0}, t_{1}, t_{2}, \ldots$ of the sum? and are they related in the classical way via some natural class DFK? The answers are not far off. Divide the line into two pieces by a cut at $x$ and let $Q^{-}\left[Q^{+}\right]$be the operator $Q$ restricted to functions on the half-line $y \leqq x[y \geqq x]$ vanishing at $x . Q^{-}$and $Q^{+}$define side spectra $\lambda_{n}^{ \pm}(n>0)$, and the divisor is simply the points $\mathfrak{p}=\left(\lambda_{n}^{+},+1\right)$ on the upper sheet of $\mathbf{K}$ and $\mathfrak{p}=\left(\lambda_{n}^{-},-1\right)$ on the lower sheet. Now regard $\omega_{n}=\left(\lambda-\lambda_{n}\right)^{-1} d \lambda(n \geqq 0)$ as a differential of the first kind. Formally,

$$
\sum \int_{\infty}^{\mathfrak{p}} \omega_{n}=\sum_{k=0}^{\infty} \int_{\lambda_{\bar{k}}}^{\lambda_{k}^{+}} \frac{d \lambda}{\lambda-\lambda_{n}}=\ell \mathrm{g} \prod_{k=0}^{\infty} \frac{\lambda_{k}^{+}-\lambda_{n}}{\lambda_{k}^{-}-\lambda_{n}}
$$

provided the side spectra are disjoint from the fixed spectrum $\lambda_{n}=2 n$. But $\lambda_{k}^{ \pm}(k \geqq 0)$ are the roots of $w_{ \pm}(x, \lambda)=0$, while $e^{-t_{n}} w_{+}(x, \lambda)=w_{-}(x, \lambda) \neq 0$ for $\lambda=\lambda_{n}$, so that

$$
\sum \int_{\infty}^{p} \omega_{n}=\ell \mathrm{g} \frac{w_{+}\left(x, \lambda_{n}\right)}{w_{-}\left(x, \lambda_{n}\right)}=-t_{n}
$$

$26 \mathfrak{p}$ is the typical point $[\lambda(\mathfrak{p}), y(\mathfrak{p})]$ of $\mathbf{K}$.

$27 p_{i}=\lambda_{j}$ is disallowed.

28 The presence of poles looks odd but their residues are really vestigial periods. 
Amplification 1. The side spectra move with $x$ as in Fig. 4: For $x=-\infty$, the lefthand spectrum is absent and $\lambda_{n}^{+}=2 n(n \geqq 0)$. These points move steadily to the right as $x$ comes in from $-\infty$; simultaneously, the left-hand spectrum enters from $+\infty$, crossing the right-hand spectrum only at the points $2,4,6$, etc. and occupying, for $x=\infty$, the place $\lambda_{n}^{-}=2 n(n \geqq 0)$, the right-hand spectrum having disappeared. The rule that the side spectra cross only at $2,4,6$, etc. follows from the fact that if, e.g., $\lambda_{1}^{+}=6=\lambda_{3}^{-}$, then $e_{3}(x)=0$, so that $e_{3}$ is also an eigenfunction of $Q^{-}$, i.e., 6 is a left-hand eigenvalue.

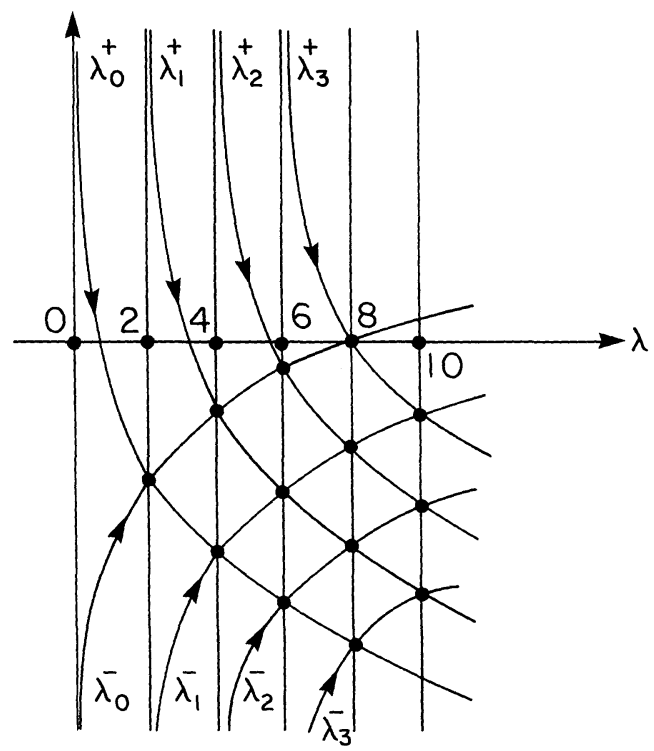

Fig. 4.

\section{Appendix: Numerical Results}

The following pictures of $q(x)=x^{2}-1-2 D^{2} \lg \theta$ were kindly made for us by O. McBryan by numerical evaluation of $\theta$ for five active parameters $t_{j}(0 \leqq j \leqq 4)$; their values are indicated under each figure. Figures 5-7 display an unexpected progressively deep well. This appears to contradict the fact that as $t=t_{0} \uparrow \infty$ in $e^{t \mathbf{X}_{0}}\left(x^{2}-1\right)=q(x)$, the final potential $q^{+}(x)$ has spectrum $\lambda_{n}^{+}=2 n(n \geqq 1)$ higher than that of $q^{0}(x)=x^{2}-1$ : actually, the well moves off to $+\infty$ and disappears at $t=\infty$. 
492

H. P. McKean and E. Trubowitz

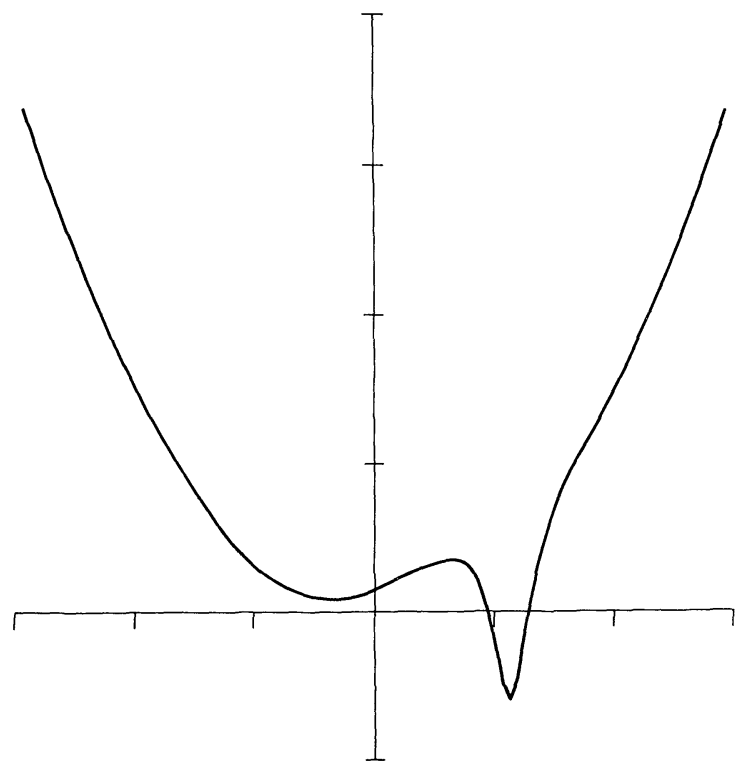

Fig. 5. $(7,0,0,0,0)$

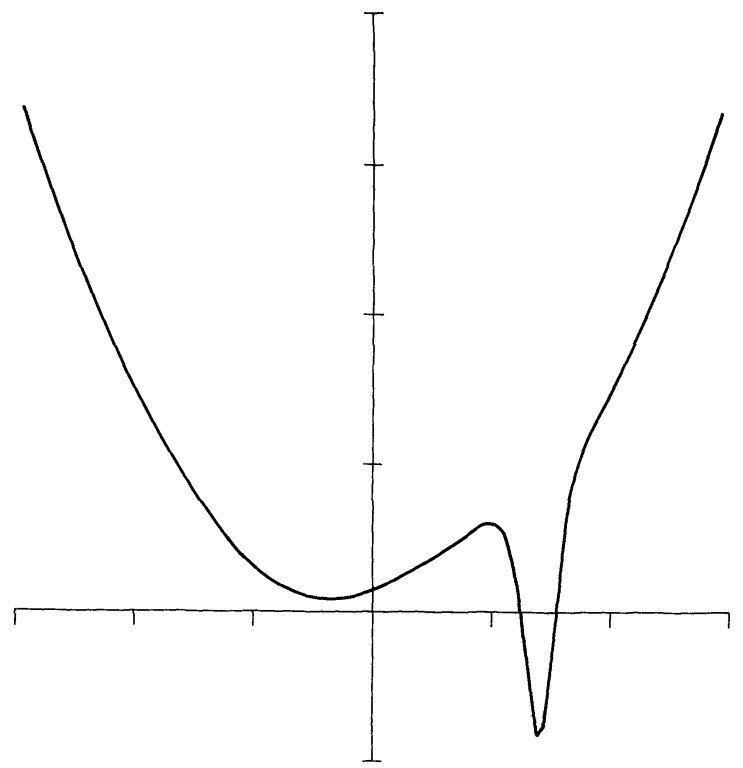

Fig. 6. $(10,0,0,0,0)$ 
Spectral Class of the Harmonic Oscillator

493

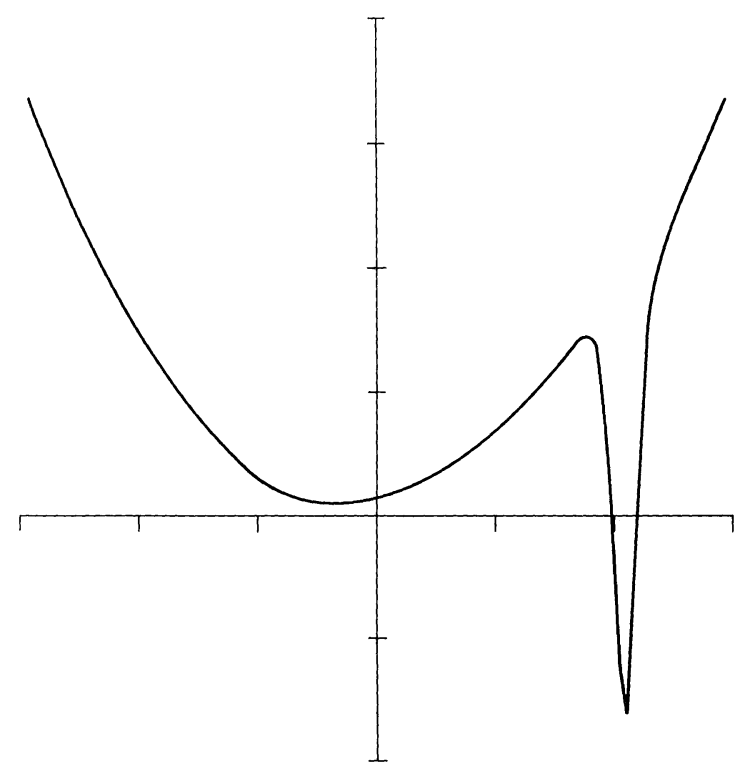

Fig. 7. $(20,0,0,0,0)$

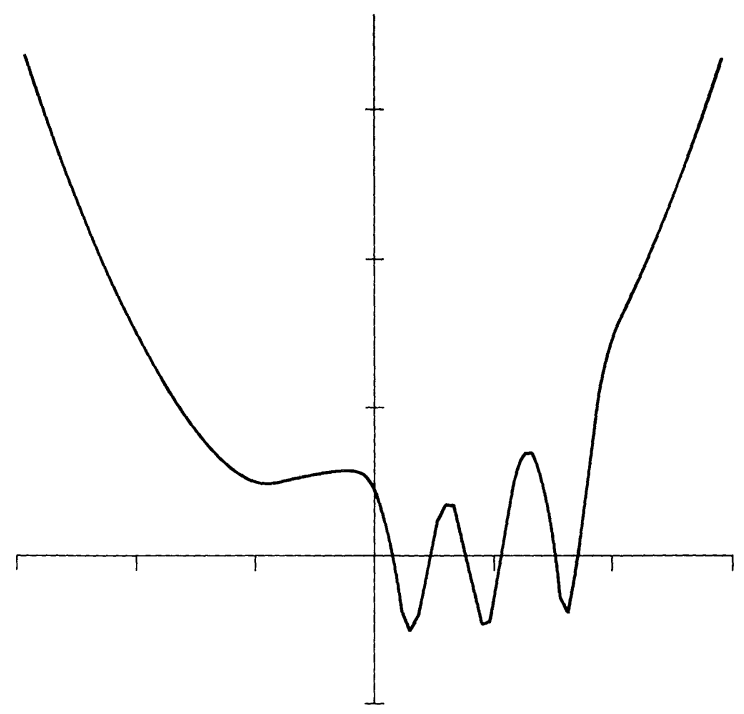

Fig. 8. $(7,7,7,0,0)$ 
494

H. P. McKean and E. Trubowitz

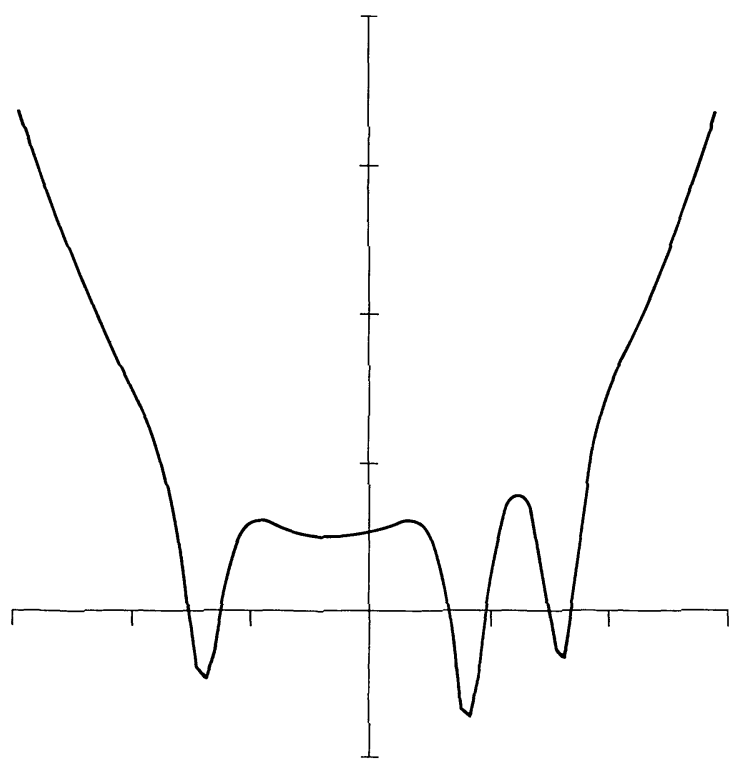

Fig. 9. $(7,-7,7,0,0)$

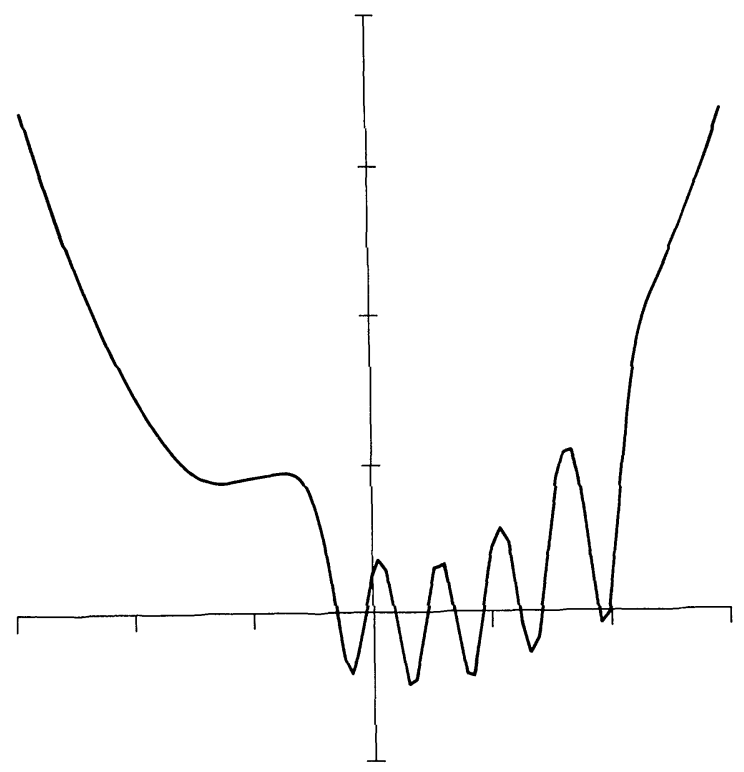

Fig. 10. $(7,7,7,7,7)$ 


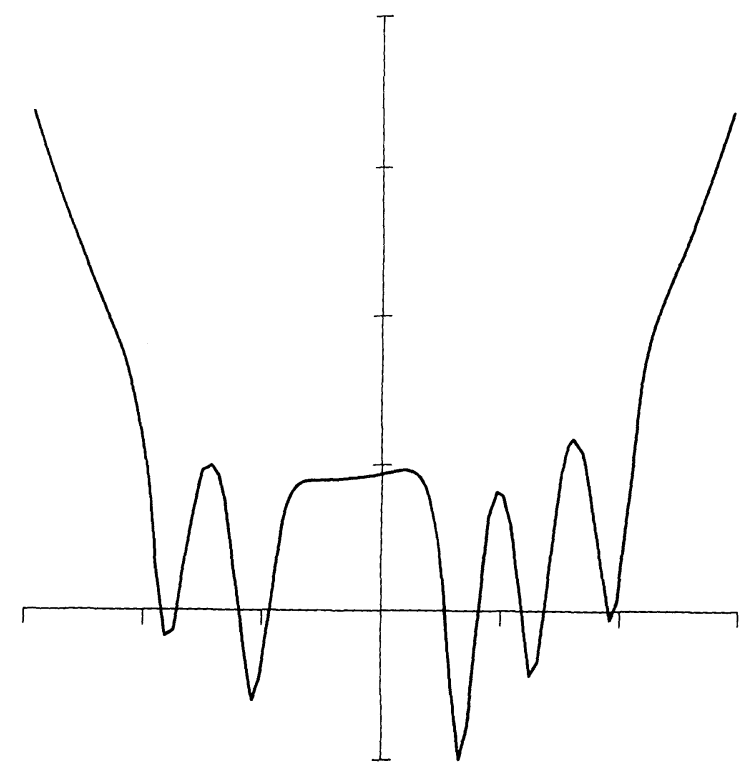

Fig. 11. $(7,-7,7,-7,7)$

Acknowledgements. It is a pleasure to thank O. McBryan for the pictures in the Appendix, P. Deift for stimulating conversations, T. Nanda for help with the Gaussian elimination of Sect. 2, and I. M. Gelfand who encouraged us to publish this account.

The authors gratefully acknowledge the support of the National Science Foundation under Grants NSF/MCS76-97036 and NSF/MCS-800-2561, respectively. H. P. McKean would also like to thank the Mathematical Institute. Oxford University, for hospitality, October 1979-June 1980, during which period this paper was put into final form.

\section{References}

Baker, H. : Abel's Theorem and the allied theory, including the theory of the theta function. Cambridge:

Cambridge Univ. Press, 1897

Bateman, H. : Higher transcendental functions (2). New York : McGraw-Hill 1953

Borg, G.: Acta Math. 78, 1-96 (1945)

Deift, P., Trubowitz, E. : CPAM 32, 121-251 (1979)

Gelfand, I. M., Levitan, B. : Izvest. Mat. Akad. Nauk 15, 309-360 (1951)

Kay, I., Moses, H.: J. Appl. Phys. 27, 1503-1508 (1956)

Levinson, N. : Math. Tidsskr. 25-30 (1949)

McKean, H. P.: Theta functions, solitons, and singular curves. Partial Differential Equations and

Geometry. C. Byrnes. (ed.) New York and Basel: M. Dekker, Inc., 1979

McKean, H. P., van Moerbeke, P. : Invent. Math. 30, 217-274 (1975)

McKean, H. P., Trubowitz, E. : BAMS 84, 1042-1085 (1978)

Communicated by A. Jaffe

Received June 1, 1981 
